\title{
Dynamical effects in electron transfer reactions. II. Numerical solution
}

\author{
Walter Nadler and R. A. Marcus \\ Arthur Amos Noyes Laboratory of Chemical Physics, ${ }^{2}$ California Institute of Technology, Pasadena, \\ California 91125
}

(Received 24 October 1986; accepted 23 December 1986)

\begin{abstract}
In part I a reaction-diffusion equation was introduced for the description of electron transfer reactions which are induced by fluctuations in both the solvent polarization and in the intramolecular vibrational coordinates. We analyze the model employing a generalized moment expansion for the time behavior of the survival probability $Q(t)$, i.e., for the fraction of molecules that have not transferred their electron at time $t$. Numerical and, in the narrow reaction window limit, analytical solutions are given for the average survival times $\tau$. When the contribution of the intramolecular coordinates is appreciable an approximate power-law behavior $\tau \propto \tau_{L}^{\alpha}$, with $0<\alpha \leqslant 1$, is found for the dependence of $\tau$ on the solvent dielectric relaxation time $\tau_{L}$, in the large $\tau_{L}$ regime. Within the framework of the generalized moment description $Q(t)$ is approximated as a superposition of several optimized exponential functions. In the small and intermediate $\tau_{L}$ regimes it is found that a single- or bi-exponential description, respectively, is sufficient. Simple formulas for such approximations in terms of the average survival times are given. Furthermore it is demonstrated that in the large $\tau_{L}$ regime a truly multiexponential time behavior for the survival probability is encountered which, over a certain range of time, can appear to be algebraic, i.e., $Q(t) \propto t^{-\gamma}$. The relation of these results to experimental data is discussed.
\end{abstract}

\section{INTRODUCTION}

The dynamical effects of solvent dielectric relaxation on the rates of electron transfer reactions have been the focus of a number of recent experimental ${ }^{1-9}$ and theoretical ${ }^{10-19}$ investigations. However, since the theoretical investigations on this topic were naturally centered on the description of the influence of the solvent, additional contributions to the electron transfer from intramolecular degrees of freedom were most often neglected. In part I of this series ${ }^{18}$ the electron transfer process was treated in a way which incorporated the effects of fluctuations of both the solvent polarization and of any intramolecular vibrational changes during the reaction. The treatment led to a diffusion equation for the solvent polarization coordinate which included a reactive term also dependent on this coordinate. In the absence of intramolecular contributions this reactive term simplifies to a delta function. A first analysis of this solvational plus intramolecular vibrational model, including an approximate description of resulting reaction rates, was given in part $I$.

In the present paper a numerical solution of the model of part $I$ is given, making use of a multiple exponential expression for the survival probability and evaluating the terms in it using the generalized moment algorithm..$^{20,21}$ To make the present paper relatively self-contained, the model is reviewed in Sec. II and the method of solution is described there in detail. In Sec. III the numerical results for mean survival times are presented and comparison is made with several approximations. Numerical results for the time dependence of the survival probability, i.e., the fraction of molecules that have not transferred their electron, are given in Sec. IV, and a comparison is made with single- and bi-expo-

\footnotetext{
a) Contribution No. 7495.
}

nential approximations. Concluding remarks, discussion, and remarks on some experiments appear in Sec. V.

\section{MODEL AND METHOD OF SOLUTION}

\section{A. Reaction-diffusion equation}

In solutions, stochastic degrees of freedom, such as a fluctuating solvent polarization coordinate $X$, as well as intramolecular vibrational degrees of freedom, may contribute to electron transfer processes. The first of these coordinates, it was argued in part I, can often be regarded as "slow," and the second as relatively "fast." In such a situation electron transfer reactions can be described by a reaction-diffusion equation. For the probability distribution $P(X, t) d X$, i.e., the fraction of molecules that have not transferred their electron at time $t$ and which experience a solvent polarization coordinate in the interval $(X, X+d X)$, this equation has the form

$$
\frac{\partial}{\partial t} P(X, t)=[\mathbf{L}(X)-k(X)] P(X, t) .
$$

$\mathbf{L}(X)$ is a Fokker-Planck operator that determines the stochastic motion along the polarization coordinate and has the form

$$
\mathbf{L}(X)=D \frac{\partial}{\partial X}\left\{\frac{\partial}{\partial X}+\beta\left[\frac{d}{d X} U(X)\right]\right\},
$$

where $D$ is the "diffusion coefficient" for the solvent fluctuations, and $U(X)$ is a potential that, for the generic case considered in part $\mathrm{I}$, is assumed to be harmonic

$$
U(X)=\frac{1}{2} X^{2}
$$

$\beta$ denotes the scaled inverse temperature, $\left(k_{B} T\right)^{-1}$.The diffusive motion along the polarization coordinate alone, i.e., the fluctuations of the solvent polarization in the absence of 
reaction, would lead to a relaxation of the distribution function $P(X, t)$ from an initial distribution $P(X, t=0)$ to an equilibrium Boltzmann distribution

$$
P_{0}(X) \propto \exp [-\beta U(X)],
$$

on a time scale given by

$$
\tau_{L}=(\beta D)^{-1} .
$$

That the stationary distribution (2.4) is not reached is due to the reactive term $k(X)$ in Eq. (2.1). It describes the electron transfer process, which is induced at each $X$ by the solvational and vibrational motion. In deriving the reactive term the assumption was made that equilibration in the vibrational coordinates is fast, that the motion across the transition state is ballistic rather than diffusive, ${ }^{22(a)}$ and, for computational simplicity, that no backreaction occurs (equations involving a back reaction are given later in this section). The reactive term has the form ${ }^{22(b)}$

$$
k(X)=v_{q} \exp [-\beta \Delta G(X)],
$$

with a frequency factor $v_{q}$ which depends on whether the crossing of the transition state is adiabatic or nonadiabatic and is described in part I. The $X$-dependent free energy barrier $\Delta G(X)$ was derived in part I to be

$$
\Delta G(X)=\frac{1}{2}\left(\frac{\lambda_{0}}{\lambda_{i}}\right)\left(X-X_{c}\right)^{2},
$$

where $\lambda_{i}$ and $\lambda_{0}$ are quantities describing the contributions from intramolecular and environmental reorganization processes, respectively, to a reorganization energy term $\lambda . X_{c}$ denotes the value of the polarization coordinate at the point of the transition state hypersurface where the intramolecular coordinates have their initial equilibrium value (compare Fig. 1 of part I).

One time scale of the reaction process is given by the thermal equilibrium expectation value of $k(X)$ :

$$
k_{e}=\langle k(X)\rangle_{0}=\int_{-\infty}^{\infty} d X k(X) P_{0}(X),
$$

where \langle\rangle$_{0}$ denotes averaging with respect to the equilibrium distribution function $P_{0}(X)$. As will be discussed below, this time scale is to be compared with $\tau_{L}$, the diffusive time scale. The rate constant $k_{e}$ has the value ${ }^{18}$

$$
k_{e}=v_{q}\left(1+\frac{\lambda_{0}}{\lambda_{i}}\right)^{-1 / 2} \exp \left(-\beta \Delta G^{*}\right),
$$

with a free energy barrier $\Delta G^{*}$ given by

$$
\Delta G^{*}=\frac{1}{2}\left(1+\frac{\lambda_{i}}{\lambda_{0}}\right)^{-1} X_{c}^{2} .
$$

$X_{c}^{2}$ is given in terms of the standard free energy of the reaction, $\Delta G^{0}$, and the reorganizational terms, $\lambda_{i}$ and $\lambda_{0}$, by comparing Eq. (2.10a) with the equivalent expression ${ }^{18}$

$$
\Delta G^{*}=\frac{\lambda}{4}\left(1+\frac{\Delta G^{0}}{\lambda}\right)^{2}
$$

with $\lambda=\lambda_{0}+\lambda_{i}$.

It is of notational convenience to consolidate the molecular parameters. By the transformation $\sqrt{\beta} X \rightarrow X$ the Fokker-Planck operator can be written in the convenient form

$$
\mathbf{L}(X)=\tau_{L}^{-1} \frac{\partial}{\partial X}\left[\frac{\partial}{\partial X}+X\right],
$$

and the Boltzmann distribution (2.4) becomes, after normalization,

$$
P_{0}(X)=\frac{1}{\sqrt{2 \pi}} e^{-(1 / 2) X^{2}}
$$

Also, by the use of Eq. (2.9) the frequency factor $v_{q}$ can be eliminated from the expression for $k(X)$ :

$$
k(X)=k_{e} \sqrt{1+\frac{\lambda_{0}}{\lambda_{i}}} \exp \left\{-\beta\left[\Delta G(X)-\Delta G^{*}\right]\right\},
$$

with

$$
\beta \Delta G(X)=\frac{1}{2}\left(\frac{\lambda_{0}}{\lambda_{i}}\right)\left(X-X_{c}\right)^{2},
$$

and $X_{c}$ related to $\beta \Delta G^{*}$ by

$$
X_{c}=\sqrt{2\left(1+\frac{\lambda_{i}}{\lambda_{0}}\right) \beta \Delta G^{*}} .
$$

Therefore, the present model depends only on the diffusive and reactive time scales, i.e., on $\tau_{L}$ and $k_{e}$, and on the reaction parameters $\lambda_{i} / \lambda_{0}$ and $\beta \Delta G^{*}$.

A quantity of experimental relevance is the fraction of reactant molecules that have not transferred their electron by time $t$, which was termed the survival probability in part I,

$$
Q(t)=\int_{-\infty}^{\infty} d X P(X, t)
$$

For the initial distribution $P(X, t=0)$ of the molecules we will assume the Boltzmann distribution (2.4) throughout this paper, ${ }^{22(c)}$ since this case appears to correspond to a common experimental situation. Due to the electron transfer processes the function $Q(t)$ decreases monotonically from 1 to 0 . Except in special cases, $Q(t)$ cannot be evaluated analytically from Eq. (2.1) and, therefore, a simplifying approximation (as in part I) or a numerical solution is needed.

There are some qualitative arguments which can be given concerning the functional form to be expected for $Q(t)$. For a large class of potentials, including the harmonic one, the operator $[\mathbf{L}(X)-k(X)]$ is expected to have a discrete spectrum of eigenvalues $-\gamma_{n}$ with $\gamma_{n}>0$ and so, in principle, a spectral expansion of $Q(t)$ can be given,

$$
Q(t)=\sum_{n=1}^{\infty} a_{n} e^{-\gamma_{n} t},
$$

with the expansion coefficients $a_{n}$ determined from the eigenfunctions and from the initial distribution $P(X, 0)$. Therefore, we should expect a multiexponential relaxation behavior for $Q(t)$ which, in certain parameter regions, may be approximated by one or two exponentials.

As has been pointed out in part $\mathrm{I}$, valuable information about the time behavior of $Q(t)$ is already given by the average survival times:

$$
\begin{aligned}
\tau_{a} & =\int_{0}^{\infty} Q(t) d t \\
\tau_{b} & =\int_{0}^{\infty} t Q(t) d t / \int_{0}^{\infty} Q(t) d t .
\end{aligned}
$$


These quantities give an estimation of the time scale for the relaxation process of $Q(t)$ and give also some hints concerning the functional form of this behavior. A comparable value of $\tau_{a}$ and $\tau_{b}$ indicates, for example, that $Q(t)$ exhibits an almost single-exponential decay, whereas strongly differing values indicate that the behavior is more complicated, e.g., bi- or multi-exponential. The quantity $\tau_{a}$ is equivalent to the mean first passage time known from diffusion controlled processes, ${ }^{23}$ and its inverse serves as one estimate for the reaction rate of the electron transfer process. The relation between $\tau_{a}$ and $\tau_{b}$ will be further delineated during the course of this paper. The quantities $\tau_{a}$ and $\tau_{b}$ have been evaluated approximately in part I. Numerical results for these quantities are obtained in the present article and are given below in Sec. III, where they are also compared with the approximate results of part $I$.

It is useful, at this stage, to review four limiting cases of Eq. (2.1) already discussed in part $I$.

\section{Fast diffusion limit, $\tau_{L} k_{\theta} \rightarrow O\left(k_{\theta} \neq 0\right)$}

In this limit the distribution of reactant molecules that experience the polarization coordinate $X$ is at all times proportional to the thermal distribution (2.4), since deviations from this form rapidly decay due to the fast diffusional relaxation processes. Therefore, the relation $P(X, t) \approx P_{0}(X) Q(t)$ holds, and with this relation one can easily derive from Eq. (2.1) that $Q(t)$ shows a single-exponential decay with $k_{e}$ as rate constant.

\section{Slow diffusion limit, $\tau_{L} k_{a} \rightarrow \infty\left(k_{0}<\infty\right)$}

In this other extreme case the distribution of the polarization coordinate $X$ seen by the reactant molecules appears to be frozen in at the initial distribution $P(X, 0)$, since practically no diffusional relaxation occurs. Furthermore, each fraction of molecules that experiences a polarization coordinate $X$ reacts with a rate constant $k(X)$. Since the time behavior of $Q(t)$ is a superposition of these different reaction processes this case yields a well-known expression

$$
Q(t)=\int_{-\infty}^{\infty} d X P_{0}(X) e^{-k(X) t} .
$$

Thus, in this limit the eigenvalues $-\gamma_{n}$ in Eq. (2.12) are so closely spaced that the multiexponential form (2.12) has become essentially an integral.

The two cases above were distinguished by the different limiting values for the ratio of the diffusive and reactive time scales, irrespective of the value for the other reaction parameters. The following two cases are, in contrast, distinguished by different limiting values for the parameter $\lambda_{i} / \lambda_{0}$, which regulates the width of the Gaussian reactive term $k(X)$ and, therefore, will be called reaction window parameter.

\section{Narrow reaction window limit, $\lambda_{1} / \lambda_{0} \rightarrow 0$}

This limit corresponds to a vanishing contribution of the intramolecular degrees of freedom to the electron transfer reaction, i.e., $\lambda_{i} \approx 0$. The reactive term now assumes the simple form of a delta function

$$
k(X)=k_{e}\left[P_{0}(X)\right]^{-1} \delta\left(X-X_{c}\right),
$$

which makes it possible to give some analytical results. For the case $k_{e} \rightarrow \infty$ and $X_{c}=0$ (i.e., $\beta \Delta G^{*}=0$ ), the form of $Q(t)$ was derived in Ref. 24 and in part I to be ${ }^{25}$

$$
Q(t)=\frac{2}{\pi} \sin ^{-1}\left(e^{-t / \tau_{L}}\right) .
$$

Analytical results for the average survival times for the other values of $k_{e}$ and $X_{c}$ are derived in the present paper.

\section{Wide reaction window limit, $\lambda_{1} / \lambda_{0} \rightarrow \infty$}

In this other extreme case of the reaction window parameter the contributions from the solvent to the electron transfer process vanish, $\lambda_{0}$ being zero. The reactive term is practically constant and equal to $k_{e}$ over the relevant range of the potential. Therefore, in this limit the time behavior of $Q(t)$ is simple again, being a single-exponential decay with $k_{e}$ as rate constant.

Before we proceed to describe the method employed in this paper to evaluate numerically the average survival times (2.13) and the survival probability $Q(t)$, we would like to discuss first the relation of the model presented here to several reaction diffusion models treated in the recent chemical physics literature. ${ }^{26}$ The physical description of the electron transfer process underlying these models has already been discussed in part I.

Reaction-diffusion models for electron transfer processes coupled to solvent polarization fluctuations without the inclusion of intramolecular degrees of freedom have been treated in several papers. ${ }^{12(a), 13,17}$ Though different approaches were employed in these investigations the models derived had quite comparable features. They resulted in the description of the transfer process as a "diffusion" of the solvent polarization (or an equivalent coordinate) along a one-dimensional free energy surface of the reactant, with the reaction occuring via the crossing over to the free energy surface of the product at the intersection point of the two free energy surfaces. In the adiabatic case the crossing over to the product free energy surface occurs with unit probability. In this case, neglecting the backreaction, the reaction process can be viewed as a diffusion in a one-dimensional potential well with an absorptive boundary at the intersection point of the two free energy surfaces. When the intersection does not pass through the minimum of the reactant's free energy surface there is a reaction barrier, and for high enough barriers the approach of $\mathrm{Kramers}^{27}$ can be employed to derive expressions for the reaction rate. ${ }^{13,17}$ In the nonadiabatic case the crossing over to the product free energy surface at the intersection point occurs with a probability of less than one and, therefore, the part of the reactant free energy surface that lies energetically higher than that of the product free energy surface can be reached. In this case, again with neglect of the back reaction, the reaction process is described by a diffusion on the reactant potential curve including a reactive term of delta-function form at the intersection point of the potentials. ${ }^{12(a)}$

The model just described for a nonadiabatic electron transfer process corresponds to the narrow reaction window limit of our model, the point $X_{\mathrm{c}}$ representing the intersection point of the two free energy surfaces. The inclusion of intra- 
molecular degrees of freedom gives rise to a broadening of the delta-function reactive term to a Gaussian.

In Ref. 15 a model was introduced where intramolecular contributions to the electron transfer process enter through the inclusion of exchange processes of vibronic quanta. This gives rise to a reactive term $k(X)$ consisting of a sum of delta functions centered at different values $X_{v}$ of the polarization coordinate $X$, the separation of successive $X_{v}$ 's being proportional to the vibration frequency $\Omega$. The respective contributions of the various delta functions are given by Franck-Condon factors. The present model can be regarded as the classical analog of the semi-quantum-mechanical model presented in Ref. 15. Indeed, Boltzmann-weighted Franck-Condon factors assume the form of a Gaussian distribution in the continuum limit. The models discussed in Refs. 12(b), 12(c), and 14, are also classical analogs of Ref. 15. They correspond to the present model, in case the back reaction is neglected, with a similar $k(X)$ in case of Ref. 14 and $k(X)$ unspecified in case of Refs. 12(b) and 12(c).

An extension of the present model to include the backreaction is straightforward. One has to take into account the product distribution $P^{(p)}(X, t) d X$, i.e., the fraction of molecules that have already transferred their electron and experience a solvent polarization in the interval $(X, X+d X)$, in addition to the reactant distribution $P(X, t) d X$. Equation (2.1) is then replaced by the coupled equations ${ }^{28}$

$$
\begin{aligned}
\frac{\partial}{\partial t} P(X, t)= & {[\mathbf{L}(X)-k(X)] P(X, t) } \\
+ & k^{(p)}(X) P^{(p)}(X, t), \\
\frac{\partial}{\partial t} P^{(p)}(X, t)= & {\left[\mathbf{L}^{(p)}(X)-k^{(p)}(X)\right] } \\
& \times P^{(p)}(X, t)+k(X) P(X, t),
\end{aligned}
$$

where $\mathbf{L}^{(p)}(X)$ is given by Eq. (2.2), with the potential $U(X)$ replaced by the product potential $U^{(p)}(X) ; k^{(p)}(X)$ is the $X$-dependent reactive term for the back reaction which was neglected in part I and in Eq. (2.1). Equations (2.17) have to be solved subject to appropriate initial conditions, e.g., $P^{(p)}(X, t=0)=0$ and a Boltzmann distribution for $P(X, 0)$.

For simplicity of presentation, such an extension was not considered in part I and will not be considered here. However, it can be readily treated, and we shall do so in a later publication. We may note, although, that we do not expect that the results will change qualitatively. As was already discussed in part $I$, the inclusion of the backreaction effect typically changes the resulting rate for equilibration by a factor of two or less when $k(X) \geqslant k^{(p)}(X)$, which is usually the case in experiments.

In closing this discussion we may note that reactiondiffusion models which correspond to the model of the present paper have also been applied in physical situations that are quite different from the above discussed electron transfer problems. The narrow reaction window limit of our model for the parameter values $X_{c}=0$ and $k_{e} \rightarrow \infty$ was treated analytically by Schulten et $a l .{ }^{24}$ in the context of protein folding. A reaction-diffusion equation with a functional form similar to our model was derived by Agmon and Hopfield ${ }^{29(b)}$ for the description of the anomalous binding behavior of $\mathrm{CO}$ to the heme group in myoglobin. They treated their model by both direct numerical integration of Eq. (2.1) and by calculating the spectral expansion (2.12) numerically. Numerical values for the average survival time $\tau_{a}$ have been obtained by them by a method ${ }^{29(a)}$ somewhat similar to ours, to be presented in Sec. II C. Bagchi et al. ${ }^{30}$ used a similar equation for the description of electronic relaxation processes in solvents. They employed an expansion in a set of orthogonal functions for their analysis.

We shall use here a different approach, based on the generalized moment expansion of the survival probability $Q(t)$.

\section{B. Generalized moment expansion}

To construct an approximation to $Q(t)$ we start, as in I, with the Laplace transform

$$
\bar{Q}(s)=\int_{0}^{\infty} d t e^{-s t} Q(t) .
$$

In Ref. 21 it has been demonstrated that, by the use of the adjoint Fokker-Planck operator, which has the form

$$
\mathbf{L}^{+}(X)=\tau_{L}^{-1}\left[\frac{\partial}{\partial X}-X\right] \frac{\partial}{\partial X},
$$

for $\mathbf{L}(X)$ given by Eq. $\left(2.2^{\prime}\right)$, the Laplace transform of many observables can be written as the matrix element of a nonHermitian resolvent operator. In our case, this matrix element has the form

$$
\bar{Q}(s)=\left\langle 1\left|\left[s-\mathbf{L}^{+}(X)+k(X)\right]^{-1}\right| 1\right\rangle_{0},
$$

where $\langle\mid\rangle_{0}$ denotes a scalar product with $P_{0}(X)$ as weight function, ${ }^{31}$

$$
\langle U \mid V\rangle_{0}=\int_{-\infty}^{\infty} d X P_{0}(X) U(X) V(X),
$$

and 1 in Eq. (2.20) is the unit function which represents the operand of the resolvent operator $\left[s-\mathbf{L}^{+}(X)+k(X)\right]^{-1}$.

$\bar{Q}(s)$ has the following asymptotic expansions for high and low frequencies:

$$
\begin{aligned}
& \bar{Q}(s) \sim s^{-1} \sum_{n=0}^{\infty}(-s)^{-n} \mu_{n} \text { for } s \rightarrow \infty, \\
& \bar{Q}(s) \sim \sum_{n=0}^{\infty}(-s)^{n} \mu_{-n-1} \text { for } s \rightarrow 0,
\end{aligned}
$$

with the expansion coefficients, the generalized moments, given by

$$
\mu_{n}=\left\langle 1\left|\left[k(X)-\mathbf{L}^{+}(X)\right]^{n}\right| 1\right\rangle_{0} .
$$

These generalized moments determine the high- and lowfrequency behavior of $\bar{Q}(s)$, i.e., the short- and long-time behavior of $Q(t)$. Once the $\mu_{n}$ are known they can be used for the construction of an approximation $\bar{q}(\mathrm{~s})$ to $\bar{Q}(s)$. In our case, since we are interested in the numerically correct description of the behavior of $Q(t)$ over the whole time range, such an approximation $\bar{q}(s)$ should be designed to reproduce the correct high-frequency as well as the correct low-frequency behavior of $\bar{Q}(s)$ in a balanced way. We shall, therefore, require that the approximation $\bar{q}(s)$ of order $N$ reproduces $N$ low-frequency and $N$ high-frequency moments. As functional form for $\bar{q}(s)$ we choose a sum of $N$ Lorentzians, 


$$
\bar{q}(s)=\sum_{n=1}^{N} f_{n}\left(s+\tau_{n}^{-1}\right)^{-1},
$$

which leads, in the time domain, to a sum of $N$ exponentials,

$$
q(t)=\sum_{n=1}^{N} f_{n} e^{-t / \tau_{n}}
$$

The sum of exponentials ( $2.23 \mathrm{~b}$ ) is, therefore, optimized in the sense that $q(t)$ gives the correct short- and long-time behavior of the exact function $Q(t)$. Typically, the number of exponentials needed to adequately represent $Q(t)$ is much less than needed by using a spectral expansion (2.12).

The functional form (2.23a) of $\bar{q}(s)$ together with the requirement of the correct asymptotic behavior in the limits $s \rightarrow 0$ and $s \rightarrow \infty$ makes it a two-point $[N-1, N]$-Pade approximant ${ }^{32}$ to $\bar{Q}(s)$. From Eqs. (2.22) and (2.23) it follows that the parameters $f_{n}$ and $\tau_{n}$ are solutions of the equations

$$
\sum_{n=1}^{N} f_{n} \tau_{n}{ }^{m}=\mu_{-m}, \quad m=-N,-N+1, \ldots, N-1 .
$$

An actual algorithm for the solution of these nonlinear equations for $\tau_{n}$ and $f_{n}$ is given, e.g., in Refs. 20 and 33. However, for $N=1$ and $N=2$ solutions to Eq. (2.24) can be found quite easily.

The single- and bi-exponential descriptions of $Q(t)$ are of particular interest since they are determined solely by $k_{e}$, $\tau_{a}$, and $\tau_{b}$. This can be seen from the fact that the first highand low-frequency moments have the following representations:

$$
\begin{aligned}
& \mu_{1}=\langle k(X)\rangle_{0}=k_{e}, \\
& \mu_{0}=\langle 1\rangle_{0}=1, \\
& \mu_{-1}=\int_{0}^{\infty} Q(t) d t=\tau_{a}, \\
& \mu_{-2}=\int_{0}^{\infty} t Q(t) d t=\tau_{a} \tau_{b} .
\end{aligned}
$$

We note that the average survival times $\tau_{a}$ and $\tau_{b}$ are given by $\mu_{-1}$ and $\mu_{-2} / \mu_{-1}$, respectively, which will be employed for the determination of $\tau_{a}$ and $\tau_{b}$ in Sec. III. With the above representations of the moments the corresponding approximations $q(t)$ assume the forms

$$
q_{a}(t)=e^{-t / \tau_{a}} \text { for } N=1,
$$

and

$$
q_{2}(t)=f_{1} e^{-t / \tau_{1}}+f_{2} e^{-t / \tau_{2}} \quad \text { for } N=2,
$$

with $\tau_{1,2}$ and $f_{1,2}$ given by

$$
\begin{aligned}
\tau_{1,2}= & \frac{1}{2}\left(\tau_{a} \frac{\left(1-k_{e} \tau_{b}\right)}{\left(1-k_{e} \tau_{a}\right)}\right. \\
& \left.\mp \sqrt{\tau_{a}^{2} \frac{\left(1-k_{e} \tau_{b}\right)^{2}}{\left(1-k_{e} \tau_{a}\right)^{2}}-4 \frac{\tau_{a}\left(\tau_{a}-\tau_{b}\right)}{\left(1-k_{e} \tau_{a}\right)}}\right)
\end{aligned}
$$

and

$$
f_{1,2}= \pm \frac{\tau_{1,2}\left(1-\tau_{2,1} k_{e}\right)}{\tau_{1}-\tau_{2}}
$$

Here, the upper algebraic signs refer to $\tau_{1}$ and $f_{1}$. For $\tau_{a} \approx \tau_{b}$ it is seen immediately that $q_{2}(t)$ reduces to the single-exponential function $q_{a}(t)$. By comparing $q_{2}(t)$ and $q_{a}(t)$ with higher order approximations we will analyze in Sec. IV how good their quality is in different parameter regions.

In closing this subsection we would like to point out also that a single-exponential approximation that is solely determined by the two low-frequency moments $\mu_{-1}$ and $\mu_{-2}$ leads to $\tau_{b}$ as the relaxation time. The resulting approximation

$$
q_{b}(t)=\frac{\tau_{a}}{\tau_{b}} e^{-t / \tau_{b}}
$$

is, therefore, expected to describe the asymptotic long-time behavior of $Q(t)$ correctly.

\section{Numerical determination of the generalized moments}

For the numerical evaluation of the generalized moments $\mu_{n}$ given by Eq. (2.22c) it is convenient to transform the Fokker-Planck operator $\left(2.2^{\prime}\right)$ or its adjoint into a Hermitian, symmetric form. ${ }^{34}$ This Hermitian operator $\mathbf{L}^{(s)}(X)$ [which is equivalent to the operator $-\mathbf{H}(X)$ in part $\mathrm{I}$ ] is related to $\mathbf{L}(X)$ and $\mathbf{L}^{+}(X)$ in the following way:

$\mathbf{L}^{(s)}(X)=p_{0}^{-1}(X) \mathbf{L}(X) p_{0}(X)=p_{0}(X) \mathbf{L}^{+}(X) p_{0}^{-1}(X)$,

where we have used the square root of $P_{0}(X)$,

$$
p_{0}(X)=\sqrt{P_{0}(X)},
$$

as a transformation operator. With the operator $L^{(s)}(X)$, the matrix element in Eq. (2.20) can be rewritten as the matrix element of a Schrödinger operator:

$$
\mu_{n}=\left\langle p_{0}(X)\left|\left[k(X)-\mathbf{L}^{(s)}(X)\right]^{n}\right| p_{0}(X)\right\rangle .
$$

$\langle\mid\rangle$ is now the usual scalar product with a constant weight function equal to 1 .

The next step is the introduction of auxiliary functions $\mu_{n}(X)$ defined by

$$
\mu_{n}(X)=\left[k(X)-\mathbf{L}^{(s)}(X)\right]^{n} p_{0}(X) .
$$

Once these auxiliary functions are known, the moments $\mu_{n}$ can be calculated from them by evaluation of the scalar products

$$
\begin{aligned}
& \mu_{2 n}=\left\langle\mu_{n}(X) \mid \mu_{n}(X)\right\rangle, \\
& \mu_{2 n-1}=\left\langle\mu_{n-1}(X) \mid \mu_{n}(X)\right\rangle .
\end{aligned}
$$

In particular, the moments $\mu_{-1}$ and $\mu_{-2}$ can both be determined from the single auxiliary function $\mu_{-1}(X)$ through

$$
\begin{aligned}
& \mu_{-1}=\left\langle p_{0}(X) \mid \mu_{-1}(X)\right\rangle, \\
& \mu_{-2}=\left\langle\mu_{-1}(X) \mid \mu_{-1}(X)\right\rangle .
\end{aligned}
$$

The determination of the auxiliary functions Eq. (2.32) is straightforward in the case of high-frequency moments, i.e., for $n \geqslant 0$. One simply operates successively on the functions $\mu_{n-1}(X), n>0$ :

$$
\mu_{n}(X)=\left[k(X)-\mathbf{L}^{(s)}(X)\right] \mu_{n-1}(X),
$$

beginning with $\mu_{0}(X)=p_{0}(X)$, and thereby iteratively creating the high-frequency auxiliary functions of ever increas- 
ing order $n$. For the low-frequency auxiliary functions, i.e., for $n<0$, multiplication of Eq. (2.32) on the left with the operator $k(X)-\mathbf{L}^{(s)}(X)$ results in the following differential equations for the $\mu_{-n}(X)$ :

$$
\left[k(X)-\mathbf{L}^{(s)}(X)\right] \mu_{-n}(X)=\mu_{-(n-1)}(X), \quad n>0,
$$

which have to be supplied with appropriate boundary conditions $^{21}$ in our case $p_{0}(X)(d / d X)\left[\mu_{-n}(X) / p_{0}(X)\right] \rightarrow 0$ as $X \rightarrow \pm \infty$ \}. By iteratively solving these equations, again beginning with $\mu_{0}(X)=p_{0}(X)$ as right-hand side, we can calculate the low-frequency auxiliary functions of ever increasing order $-n$.

In most cases Eqs. (2.35) cannot be solved analytically. An exception is the narrow reaction window limit and it is treated in Appendix A. However, Eqs. (2.35) can be solved numerically and for this task we employ a particular discretization procedure ${ }^{21,29(a)}$ which was justified mathematically in Ref. 21.

We first limit the infinite diffusion space to a finite inter$\operatorname{val}\left[X_{\min }, X_{\max }\right]$ where $X_{\min }$ and $X_{\max }$ are chosen so that the relevant parts of the stationary distribution $P_{0}(X)$ and of the reactive terms $k(X)$ are included. In the cases we considered a choice $X_{\max }=-X_{\min }=n_{x} \sqrt{\left\langle X^{2}\right\rangle_{0}}$, with $n_{x}$ ranging from 4 to 7 , was sufficient, in general. In each case a check was made to determine whether the numerical results are, in effect, independent of $n_{x}$.

Next, this finite diffusion space is discretized into $N_{x}$ cells. Inside those cells the representative discretization points are chosen through

$$
X(i)=X_{\min }+\left(i-\frac{1}{2}\right) \delta, \quad i=1, \ldots, N_{x},
$$

with $\delta$ being the discretization length, $\delta=\left(X_{\max }-X_{\min }\right) /$ $N_{x}$. In our applications we have found that a choice of $N_{x}$ in the range from $50 n_{x}$ to $100 n_{x}$ was sufficient to produce results independent of the step size. Again, the results were checked that they became, in effect, independent of this choice of $N_{x}$.

In a diffusion space discretized in this manner, the differential operator $\mathbf{L}^{(s)}(X)$ becomes a symmetric matrix operator $\mathbf{L}^{(s)}$ of the following tridiagonal form ${ }^{21}$ :

$\mathbf{L}_{i j}^{(s)}=\left\{\begin{array}{l}\tau_{\delta}^{-1} \text { for } i=j \pm 1 \\ -[W(i \rightarrow i+1)+W(i \rightarrow i-1)] \text { for } i=j, \\ 0 \quad \text { for all other } i, j\end{array}\right.$

with $\tau_{\delta}$ being the time scale of the discretized operator, $\tau_{\delta}$ $=\delta^{2} \tau_{L} . W(i \rightarrow j)$ is a "transition rate" between cells:

$$
W(i \rightarrow j)=\tau_{\delta}^{-1} \frac{p_{0}[X(j)]}{p_{0}[X(i)]} .
$$

In an analogous way the reactive term in Eq. (2.35) becomes a diagonal matrix, $\mathbf{K}$, with elements

$$
K_{i j}=k[X(i)] \delta_{i j} .
$$

Therefore, Eqs. (2.34) and (2.35) for the generations of the high- and low-frequency auxiliary functions become linear algebraic equations $(n>1)$ :

$$
\boldsymbol{\mu}_{n}=\left[\mathbf{K}-\mathbf{L}^{(s)}\right] \boldsymbol{\mu}_{n-1},
$$

$$
\left[\mathbf{K}-\mathbf{L}^{(s)}\right] \boldsymbol{\mu}_{-n}=\boldsymbol{\mu}_{-(n-1)},
$$

where the functions $\mu_{n}(X)$ have been replaced by vectors $\mu_{n}$ through

$$
\left(\mu_{n}\right)_{i}=\mu_{n}[X(i)] \text {. }
$$

Because of the simple tridiagonal form of $L^{(s)}$, the linear equations $\left(2.35^{\prime}\right)$ for the discretized low-frequency auxiliary functions $\mu_{-n}$ can now be solved easily using a Gaussian elimination procedure. ${ }^{35}$ In addition, Eq. (2.34') also provides an effective alternative way for the numerical determination of the discretized high-frequency auxiliary functions $\mu_{n}$. For the final evaluation of the moments the scalar products (2.33) are evaluated simply as scalar products of the vectors $\mu_{n}$.

\section{RESULTS ON AVERAGE SURVIVAL TIMES}

In the present section numerical results for the average survival times $\tau_{a}$ and $\tau_{b}$ are presented. The results were calculated with the algorithm described above, i.e., the generalized moments $\mu_{-1}$ and $\mu_{-2}$ were evaluated numerically according to Sec. II C, and from Eqs. (2.25c) and (2.25d) $\tau_{a}$ and $\tau_{b}$ follow.

Figures 1 to 3 cover the same parameter and time range
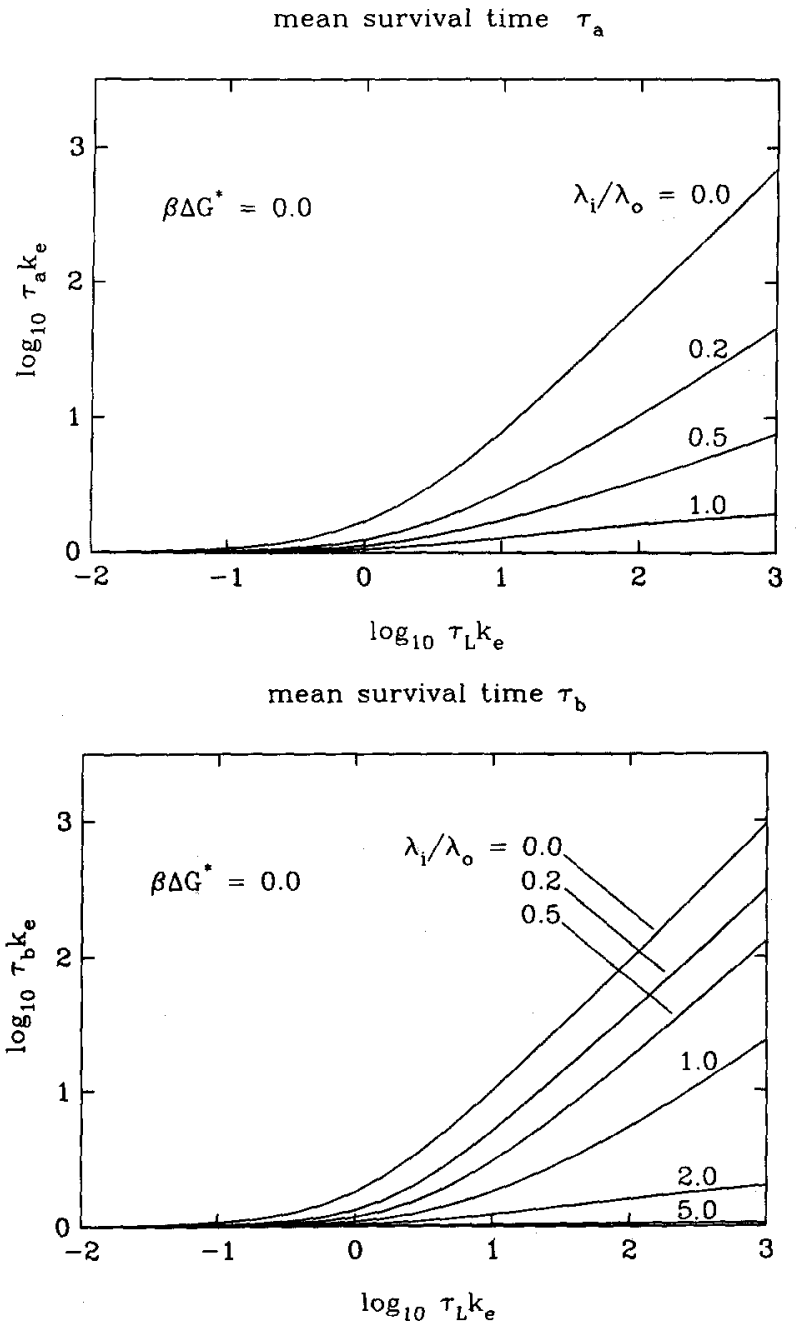

FIG. 1. Average survival time $\tau_{a}$ (a) and $\tau_{b}$ (b) vs solvent polarization relaxation time $\tau_{L}$ for various values of the reaction window parameter $\lambda_{i} / \lambda_{0}$. The reaction barrier parameter is $\beta \Delta G^{*}=0$. 
mean survival time $\tau_{\mathrm{a}}$

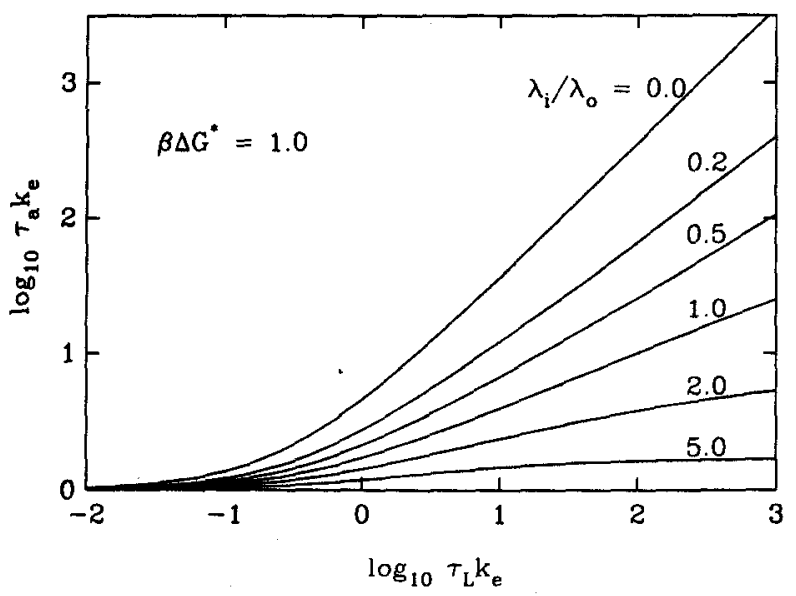

mean survival time $\tau_{b}$

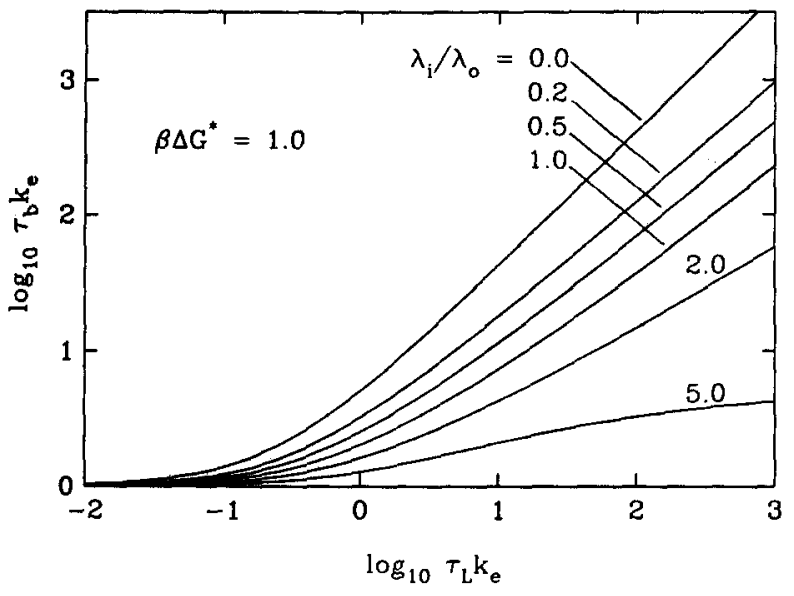

FIG. 2. Same as Fig. 1 for $\beta \Delta G^{*}=1$.

as the approximate results of part I and, to facilitate comparison, are given in the same scale. The approximation in part I agrees quite well with our numerical results in the fast diffusion regime $\left(\tau_{L} k_{e} \ll 1\right)$, in the intermediate diffusion regime $\left(\tau_{L} k_{e} \approx 1\right)$, and when the reaction window is narrow $\left(\lambda_{i} / \lambda_{0} \approx 0\right)$. For large values of $\lambda_{i} / \lambda_{0}$ the agreement in the slow diffusion regime $\left(\tau_{L} k_{e} \gg 1\right)$ is not good. ${ }^{36}$

It is useful to consider analytically the behavior for large values of the reaction window parameter $\lambda_{i} / \lambda_{0}$. In particular, for $\lambda_{i} / \lambda_{0}>1$ in case of $\tau_{a}$ and for $\lambda_{i} / \lambda_{0}>2$ in case of $\tau_{b}$, respectively, the average survival times can be shown straightforwardly to assume finite values in the slow diffusion limit. These limiting values are given by expectation values of the inverse reactive term:

$$
\begin{aligned}
\tau_{a}(\infty)= & \left\langle[k(X)]^{-1}\right\rangle_{0} \\
= & k_{e}^{-1} \sqrt{\frac{\left(\lambda_{i} / \lambda_{0}\right)^{2}}{\left(\lambda_{i} / \lambda_{0}\right)^{2}-1}} \\
& \times \exp \left[\frac{2 \beta \Delta G *}{\lambda_{i} / \lambda_{0}-1}\right] \text { for } \lambda_{i} / \lambda_{0}>1, \\
\tau_{b}(\infty)= & \frac{\left\langle[k(X)]^{-2}\right\rangle_{0}}{\left\langle[k(X)]^{-1}\right\rangle_{0}}
\end{aligned}
$$
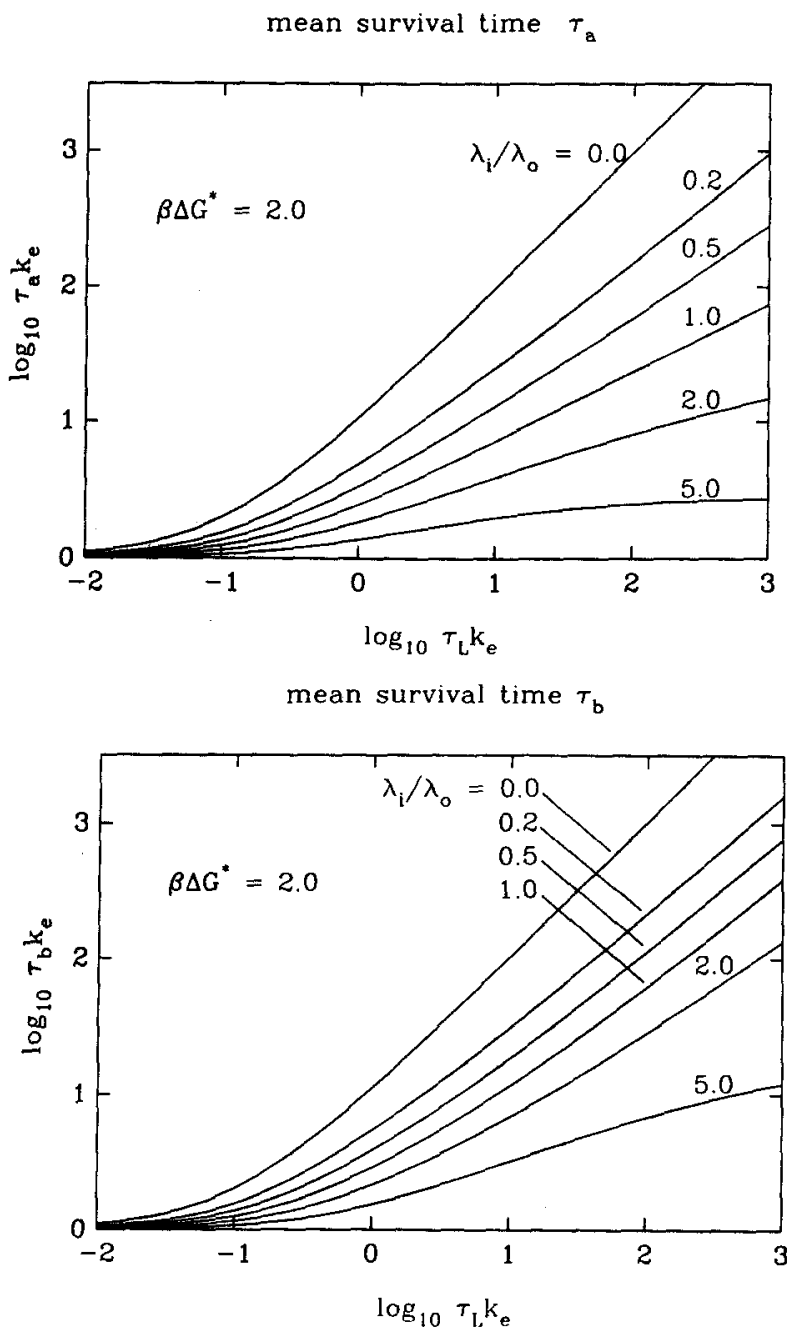

FIG. 3. Same as Fig. 1 for $\beta \Delta G^{*}=2$.

$$
\begin{aligned}
= & k_{e}^{-1} \sqrt{\frac{\left(\lambda_{i} / \lambda_{0}\right)\left(\lambda_{i} / \lambda_{0}-1\right)}{\left(\lambda_{i} / \lambda_{0}-2\right)\left(\lambda_{i} / \lambda_{0}+1\right)}} \\
& \times \exp \left[\frac{4\left(\lambda_{i} / \lambda_{0}-\frac{1}{2}\right) \beta \Delta G^{*}}{\left(\lambda_{i} / \lambda_{0}-2\right)\left(\lambda_{i} / \lambda_{0}-1\right)}\right] \text { for } \lambda_{i} / \lambda_{0}>2 .
\end{aligned}
$$

For smaller values of the reaction window parameter $\lambda_{i} / \lambda_{0}$ these expressions are seen not to be defined, and the average survival times then indeed approach infinity rather than a finite value when $\tau_{L} k_{e} \rightarrow \infty$.

That the average survival times approach a finite value as $\tau_{L} k_{e} \rightarrow \infty$ is seen, for example, in the curves for $\lambda_{i} / \lambda_{0}$ $=5.0$ in Figs. 1 (b), 2, and 3. To illustrate this limiting behavior more fully, and to illustrate also the behavior described by Eq. (3.2) below, the results corresponding to the parameters of Figs. 1 to 3 are plotted over an extended time regime in Figs. 4 to 6 . In these figures the curves for $\lambda_{i} / \lambda_{0}$ $=2.0$ in case of $\tau_{a}$, and for $\lambda_{i} / \lambda_{0}=5.0$ in case of $\tau_{a}$ and $\tau_{b}$ convincingly show the limiting behavior, Eq. (3.1).

At $\lambda_{i} / \lambda_{0}=0$, on the other hand, one sees that the log$\log$ plots of $\tau_{a}$ and $\tau_{b}$ vs $\tau_{L}$ in Figs. 1 to 6 (and later in Fig. 7) show a slope of unity. In the intervening region, $0<\lambda_{i} / \lambda_{0}$ 
mean survival time $T_{\text {a }}$

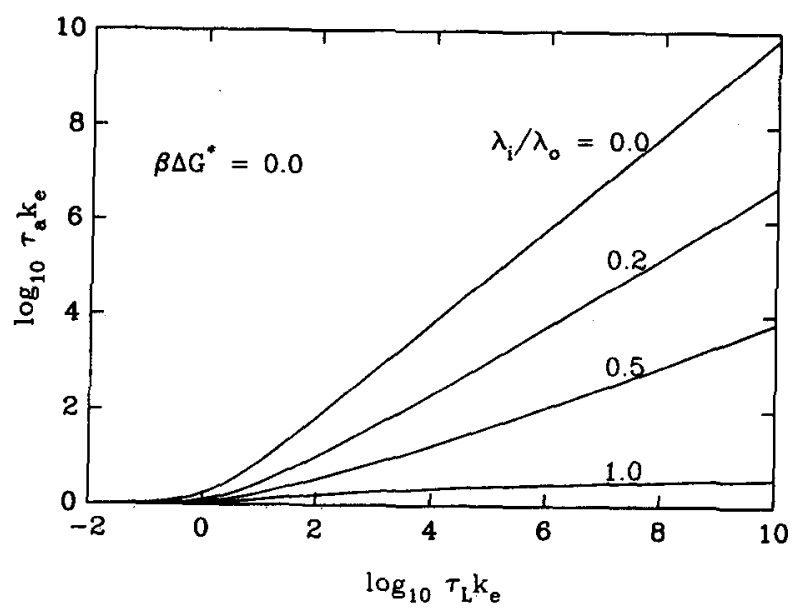

mean survival time $\tau_{\mathrm{b}}$

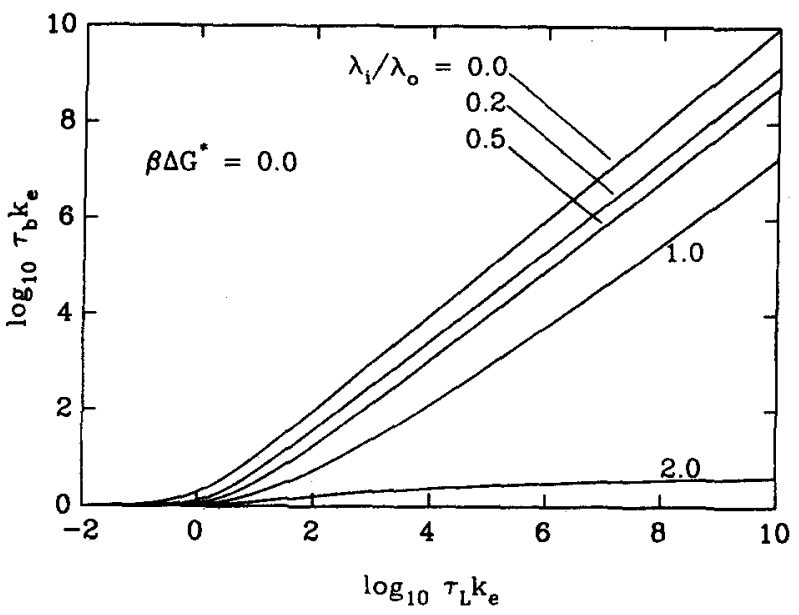

FIG. 4. Same as Fig. 1 for an extended range of $\tau_{L}$.

$<1$ for $\tau_{a}$ and $0<\lambda_{i} / \lambda_{0}<2$ for $\tau_{b}$, one would expect that the limiting $\log -\log$ plots exhibit a slope between zero and unity, corresponding to a power-law dependence,

$$
\tau_{a} \propto \tau_{L}^{\alpha_{a}} \quad\left(0<\alpha_{a}<1\right),
$$

and

$$
\tau_{b} \propto \tau_{L}^{\alpha_{b}} \quad\left(0<\alpha_{b}<1\right),
$$

of the average survival times on the solvent relaxation time $\tau_{L}$ in the slow diffusion regime $\left(\tau_{L} \rightarrow \infty\right)$. Figures 4 to 6 show that this holds to a very good degree. We note also that the apparent value for the exponent depends on the range of values for $\tau_{L} k_{e}$ under consideration. In Tables I and II asymptotic numerical values are given for the exponents $\alpha_{a}$ and $\alpha_{b}$ for different values of reaction window parameter $\lambda_{i} / \lambda_{0}$ and of the reaction barrier parameter $\beta \Delta G^{*}$. In each case $\alpha$ becomes unity in the narrow reaction window limit.

This numerical result for solvents that are characterized by a single polarization relaxation time $\tau_{L}$, namely that the inclusion of intramolecular coordinates causes the average
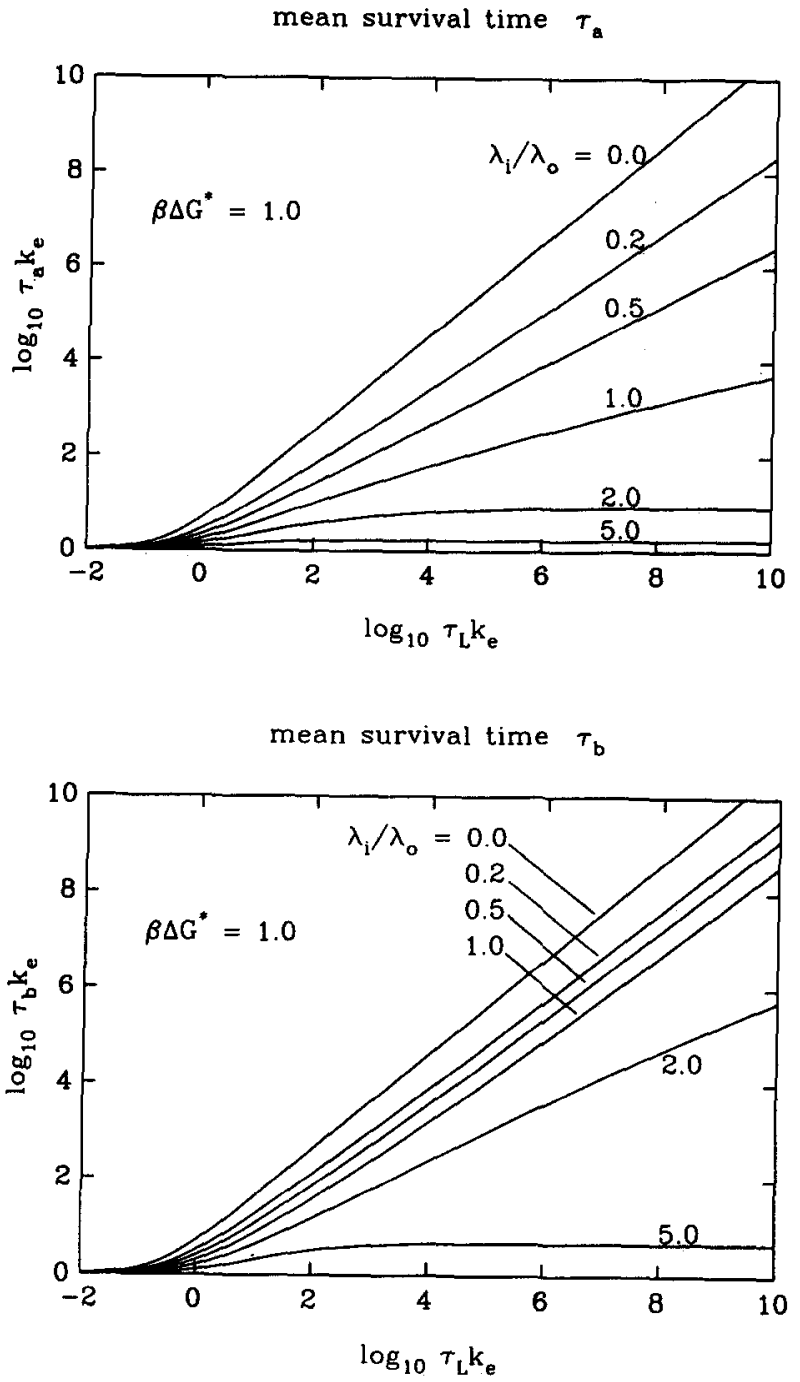

FIG. 5. Same as Fig. 2 for an extended range of $\tau_{L}$.

survival times $\tau$ to depend on $\tau_{L}$ approximately in an algebraic form (3.2), is particularly interesting. It deviates strongly from the simple $\tau \propto \tau_{L}$ behavior that results from the usual models ${ }^{12(\mathrm{a}), 13,17}$ in which electron transfer reaction processes have no contribution from intramolecular degrees of freedom. It is also different from the results in Refs. $12(\mathrm{~b}), 12$ (c), 14, and 15, where intramolecular contributions were considered. There, only limiting cases were treated that led also to a simple $\tau \propto \tau_{L}$ behavior in the large $\tau_{L}$ regime, corresponding to the behavior we found in our model for the narrow reaction window limit.

In the fast diffusion region, $\tau_{L} k_{e} \ll 1$, the moments $\mu_{-n}$ can be formally expanded in a power series in the inverse Fokker-Planck operator (a detailed derivation is given in Ref. 37),

$$
\begin{aligned}
\mu_{-1}= & k_{e}^{-1}-k_{e}^{-2} \\
& \times\left\langle 1\left|\delta k(X)\left[L^{+}(X)\right]^{-1} \delta k(X)\right| 1\right\rangle+O\left(\tau_{L}^{2}\right),
\end{aligned}
$$


mean survival time $\tau_{\text {a }}$

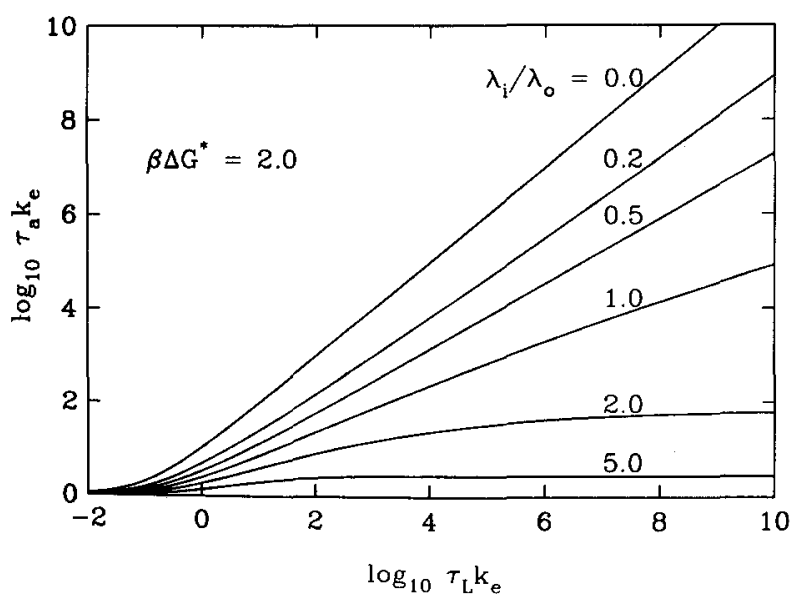

mean survival time $\tau_{b}$

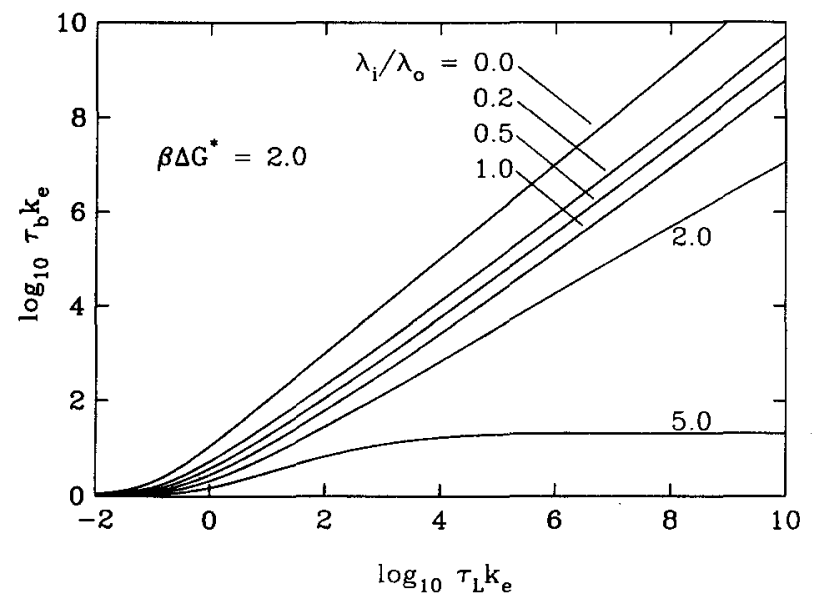

FIG. 6. Same as Fig. 3 for an extended range of $\tau_{L}$.

$$
\begin{aligned}
\boldsymbol{\mu}_{-2}= & k_{e^{-2}-2 k_{e}^{-3}} \\
& \times\left\langle 1\left|\delta k(X)\left[\mathbf{L}^{+}(X)\right]^{-1} \delta k(X)\right| 1\right\rangle+O\left(\tau_{L}^{2}\right) .
\end{aligned}
$$

Since $L^{+}(X) \propto \tau_{L}^{-1}$, such a series corresponds to an expan-

TABLE I. Asymptotic values ${ }^{\mathrm{a}}$ for the anomalous exponents $\alpha_{a}$.

\begin{tabular}{llll}
\hline \hline$\lambda_{i} \beta \Delta \lambda_{0}^{*}$ & 0 & 1 & 2 \\
\hline 0.0 & & & \\
0.1 & 1.00 & 1.00 & 1.00 \\
0.2 & 0.84 & 0.90 & 0.90 \\
0.3 & 0.74 & 0.83 & 0.85 \\
0.4 & 0.64 & 0.76 & 0.79 \\
0.5 & 0.54 & 0.69 & 0.81 \\
0.6 & 0.44 & 0.62 & 0.69 \\
0.7 & 0.34 & 0.50 & 0.63 \\
0.8 & 0.24 & 0.49 & 0.57 \\
0.9 & 0.15 & 0.43 & 0.45 \\
\hline
\end{tabular}

The asymptotic values for the anomalous exponents have been determined in the region $10^{2}<\tau_{L} k_{e}<10^{10}$; approximate anomalous exponents in a region for smaller values of $\tau_{L} k_{e}$ may be smaller, see the text for a discussion.
TABLE II. Asymptotic values for the anomalous exponent $\alpha_{b}$.

\begin{tabular}{llll}
\hline \hline$\lambda_{i} / \lambda_{0} \beta \Delta G^{*}$ & 0 & 1 & 2 \\
\hline 0.0 & & & \\
0.2 & 1.00 & 1.00 & 1.00 \\
0.5 & 0.97 & 0.96 & 0.94 \\
1.0 & 0.96 & 0.95 & 0.93 \\
1.5 & 0.91 & 0.91 & 0.90 \\
& 0.87 & 0.80 & 0.39 \\
\hline \hline
\end{tabular}

See footnote a, Table I.

sion in powers of $\tau_{L}$. Equations (3.3) are well defined and there is no possibility for a singularity of the matrix element of $\left[\mathrm{L}^{+}(X)\right]^{-1}$ since the function $\delta k(X)=k(X)-k_{e}$ can be shown to lie outside the null space of $\mathbf{L}^{+}(X)$. This is discussed in detail in Appendix B. We may note that the linear approximation (3.3a) has also been derived by Weiss $^{38}$ for a system of the form (2.1) by employing the Wilemski-Fixman approximation. ${ }^{39}$ Equation (3.3a) was derived also in part I under the assumption of a single exponential decay of $Q(t)$ in the fast diffusion regime.

To first order in $\tau_{L}$ Eqs. (3.3) give the same expression for $\tau_{a}$ and $\tau_{b}$,

$$
\tau_{a, b}=k_{e}^{-1}+F\left(\lambda_{i} / \lambda_{0}, \beta \Delta G^{*}\right) \tau_{L}+O\left(\tau_{L}^{2}\right),
$$

where the function $F\left(\lambda_{i} / \lambda_{0}, \beta \Delta G^{*}\right)$ is given by the matrix element in Eq. (3.3) and has already been derived in part I to be

$$
\begin{aligned}
F\left(\lambda_{i} / \lambda_{0}, \beta \Delta G^{*}\right)= & \ln \left[2 \frac{\left(1+c^{2}\right)}{(1+c)^{2}}\right] \\
& +2 \int_{c}^{1} d x \frac{e^{\left(1-x^{2}\right) B \Delta G^{*}}-1}{1-x^{2}}
\end{aligned}
$$

with $c=\left(1+2 \lambda_{0} / \lambda_{i}\right)^{-1 / 2}$. The integral in Eq. (3.5) can be evaluated by standard numerical methods ${ }^{35}$ and numerical values for $F$ are given in Ref. 19(b).

A comparison with the numerical results for $\tau_{a}$ and $\tau_{b}$ shows that the quality of the approximation (3.4) is somewhat better in case of $\tau_{b}$. In case of $\tau_{a}$ the approximation (3.4) is valid in the narrow reaction window regime $\left(\lambda_{i} / \lambda_{0}\right.$ $\approx 0$, see also the discussion below). In the other regions of the parameter space the approximation is valid for $\tau_{a}$ within a factor of 2, giving a rule of thumb, as long as the correction term is not larger than $k_{e}^{-1}$. For larger correction terms the deviations from the exact results for $\tau_{a}$ can be significant. In case of $\tau_{b}$ the approximation is quite applicable, i.e., within a factor of 2 , in a larger range of the narrow reaction window regime (up to $\lambda_{i} / \lambda_{0} \approx 0.5$ ) for small barrier heights. Table III illustrates this point. For larger values of the barrier height parameter $\beta \Delta G *$ the deviation becomes larger in the slow diffusion regime, a feature also illustrated in this table. In general, strong deviations of the approximation (3.4) from the numerical results occur mainly in the slow diffusion regime (large $\tau_{L}$ ), as to be expected. This demonstrates also that an extension of the Wilemski-Fixman approximation to this regime can be strongly erroneous. 
TABLE III. Comparison of approximation (3.4) for $\tau_{b}$ with numerical results."

\begin{tabular}{cclllll}
\hline \hline & $\begin{array}{c}\text { Eq. (3.4) } \\
\left.\tau_{L} k_{e} / \lambda_{0}=0\right)\end{array}$ & Exact & $\begin{array}{c}\text { Eq. (3.4) } \\
\left(\lambda_{i} / \lambda_{0}=0.2\right)\end{array}$ & Exact & $\begin{array}{c}\text { Eq. (3.4) } \\
\left(\lambda_{i} / \lambda_{0}=0.5\right)\end{array}$ & Exact \\
\hline$\beta \Delta G^{*}=0$ & & & & & & \\
1 & 1.7 & 1.9 & 1.3 & 1.3 & 1.1 & 1.2 \\
10 & 7.9 & $1.0(1)$ & 3.5 & 5.1 & 2.4 & 3.0 \\
$10^{2}$ & $7.0(1)$ & $9.5(1)$ & $2.6(1)$ & $3.8(1)$ & $1.5(1)$ & $1.8(1)$ \\
$10^{3}$ & $6.9(2)$ & $9.4(2)$ & $2.5(2)$ & $3.1(2)$ & $1.4(2)$ & $1.3(2)$ \\
$\beta \Delta G^{*}=1$ & & & & & & \\
1 & 4.6 & 5.1 & 3.1 & 3.2 & 2.5 & 2.5 \\
10 & $3.7(1)$ & $4.3(1)$ & $2.2(1)$ & $1.8(1)$ & $1.6(1)$ & $1.2(1)$ \\
$10^{2}$ & $3.6(2)$ & $4.2(2)$ & $2.1(2)$ & $1.3(2)$ & $1.6(2)$ & $7.1(1)$ \\
$10^{3}$ & $3.6(3)$ & $4.2(3)$ & $2.1(3)$ & $9.7(2)$ & $1.5(3)$ & $4.9(2)$ \\
$\beta \Delta G^{*}=2$ & & & & & & \\
1 & $1.1(1)$ & $1.1(1)$ & 6.6 & 5.4 & 4.9 & 3.8 \\
10 & $9.8(1)$ & $1.0(2)$ & $5.7(1)$ & $3.1(1)$ & $4.0(1)$ & $1.8(1)$ \\
$10^{2}$ & $9.7(2)$ & $1.0(3)$ & $5.6(2)$ & $2.1(2)$ & $3.9(2)$ & $1.1(2)$ \\
$\beta \Delta G^{*}=10$ & & & & & & \\
1 & $1.3(4)$ & $1.3(4)$ & $2.7(4)$ & $1.3(2)$ & $8.1(2)$ & $3.5(1)$ \\
\hline \hline
\end{tabular}

Numbers in parentheses indicate powers of 10 .

We have also checked the applicability of a simple [1,1]-Padé approximation

$$
\tau_{a, b} \approx \frac{F \tau_{L} \tau_{a, b}(\infty)+k_{e}^{-1}\left[\tau_{a, b}(\infty)-k_{e}^{-1}\right]}{F \tau_{L}+\left[\tau_{a, b}(\infty)-k_{e}^{-1}\right]},
$$

with $F$ from Eq. (3.5) and $\tau_{a, b}(\infty)$ the limiting values [Eq. (3.1)] of the average survival times. Equation (3.6) holds for $\lambda_{i} / \lambda_{0}>1$ in case of $\tau_{a}$ and for $\lambda_{i} / \lambda_{0}>2$ in case of $\tau_{b}$ and interpolates between the slow and the fast diffusion regime. However, in the region of intermediate values for $\tau_{L} k_{e}$, i.e., the region where the change of $\tau_{a}$ and $\tau_{b}$ is strongest, strong deviations of Eq. (3.6) from the exact results may occur, unless $\lambda_{i} / \lambda_{0}$ is large. Therefore, the use of an approximation such as (3.6) is not recommended unless $\lambda_{i} / \lambda_{0}$ is large.

In the narrow reaction window limit, $\lambda_{i} / \lambda_{0}=0$, a numerical evaluation of the moments according to Sec. II C is not possible due to the singular form (2.15) of the reactive term. However, as demonstrated in Appendix A, the derivation of analytical formulas for the moments in terms of multiple integral expressions is possible. With these results the average survival times are given by

$$
\begin{aligned}
& \tau_{a}=k_{e}^{-1}+I_{1} \tau_{L} \quad\left(\lambda_{i} / \lambda_{0}=0\right), \\
& \tau_{b}=k_{e}^{-1}+I_{1} \tau_{L}+\frac{\left(I_{2}-I_{1}^{2}\right) \tau_{L}^{2}}{k_{e}^{-1}+I_{1} \tau_{L}} \quad\left(\lambda_{i} / \lambda_{0}=0\right),
\end{aligned}
$$

with $I_{1}$ and $I_{2}$ being functions of $X_{c}$ given in Eq. (A8) and Table IV; $X_{c}$ is related to $\beta \Delta G^{*}$ through Eq. $\left(2.10^{\prime}\right)$. As also shown in Appendix A, the narrow reaction window limit of the function $F$, i.e., Eq. (3.5) with $c=0$, is identical to the term linear in $\tau_{L}$ in Eq. (3.7a), $I_{1}$. Therefore, in the narrow reaction window limit the approximation linear in $\tau_{L}$ is already correct for $\tau_{a}$ and terms of higher order in $\tau_{L}$ vanish altogether. Although such a simplification does not apply for $\tau_{b}$, the third term in Eq. (3.7b) being of second order and higher in $\tau_{L}, \tau_{b}$ is approximately linear in $\tau_{L}$ in the slow diffusion regime, however, with a different slope than $\tau_{a}$,

$$
\tau_{b} \approx k_{e}^{-1}+\left(I_{2} / I_{1}\right) \tau_{L}, \text { for } \tau_{L} \gg k_{e}^{-1} \text { and } \lambda_{i} / \lambda_{0}=0 \text {. }
$$

For large values of $X_{c}$, i.e., for large values of the reaction barrier parameter $\beta \Delta G^{*}$, the ratio $I_{2} / I_{1}$ approaches $I_{1} \mathrm{nu}$ merically, see Table IV. Therefore, for large reaction barriers $\tau_{a}$ and $\tau_{b}$ become equal when $\lambda_{i} / \lambda_{0}=0$, and a truly single-exponential relaxation of $Q(t)$ is to be expected. We consider below the case of large $\beta \Delta G^{*}$ for general values of $\lambda_{i} / \lambda_{0}$, which also exhibits a single-exponential behavior.

The curves for the narrow reaction window limit in Figs. 1 to 6 (as well as in the later figures) have been calculated from Eq. (3.7) with the values for the integral expressions $I_{1}$ and $I_{2}$ given in Table IV in Appendix A.

In Fig. 7 we include some results for high reaction barriers. In the range of $\tau_{L} k_{e}$ examined the values for $\tau_{a}$ and $\tau_{b}$ are approximately equal, and so there is a single exponential decay of form (2.25) for $Q(t)$ in this regime. We note that in this case, too, the dependence of $\tau_{a}$ on $\tau_{L}$ appears to exhibit an approximate power-law behavior (3.2) over some range of $\tau_{L} k_{e}$. It may also be noted that due to the inclusion of intramolecular coordinates the observed reaction rate constant $\tau_{a}^{-1}$ can well be some decades larger than the rate that would be expected from the barrier height alone, i.e., from $\tau^{-1} \approx \tau_{L}^{-1} \exp \left(-\beta \Delta G^{*}\right)$.

Of particular experimental interest is the dependence of the average survival times on the free energy barrier $\beta \Delta G^{*}$. This quantity is related to the standard free energy of the reaction, $\Delta G^{\circ}$, through Eq. $(2.10 \mathrm{~b})$, and $\Delta G^{0}$ can be changed experimentally at approximately constant $\lambda_{i}$ and $\lambda_{0}$ by suitable changes of the reactants. 
mean survival time $\tau_{a}$

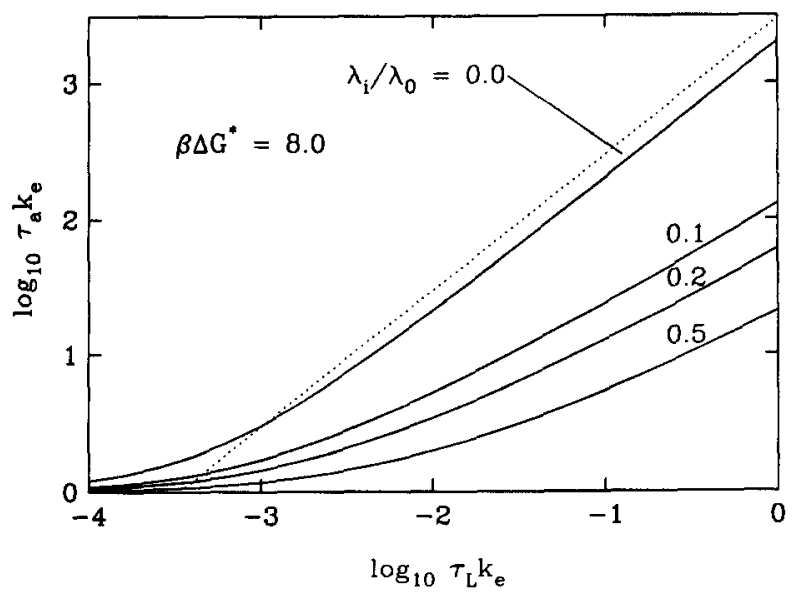

mean survival time $\tau_{\mathrm{a}}$

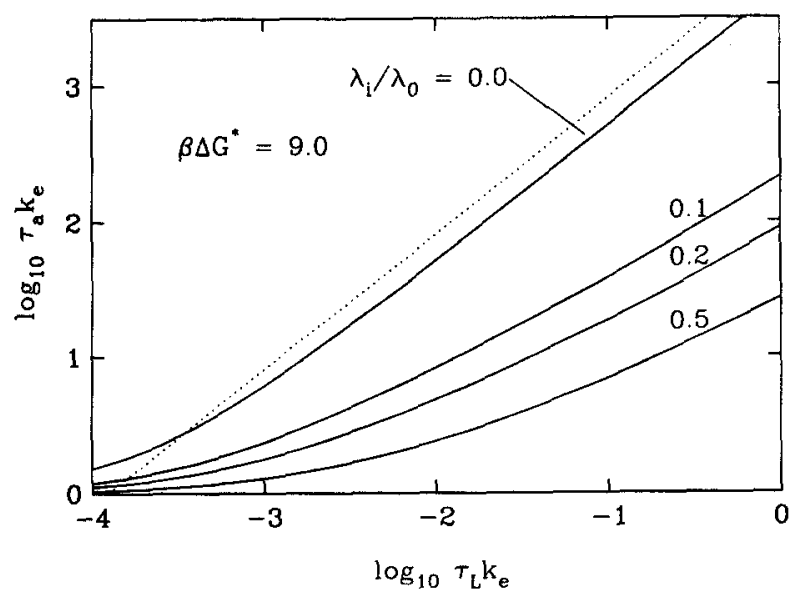

mean survival time $\tau_{\mathrm{a}}$

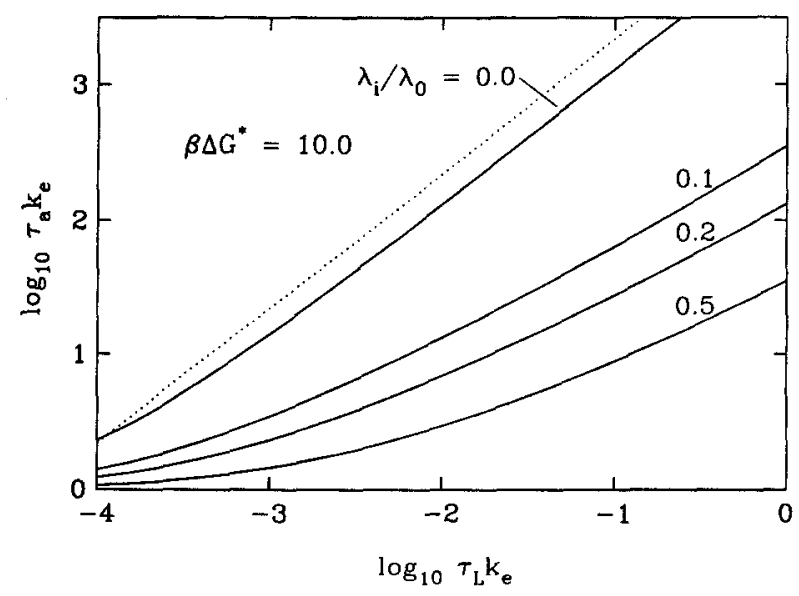

FIG. 7. Average survival time $\tau_{a}$ vs solvent polarization relaxation time $\tau_{L}$ for several values of the reaction window parameter $\lambda_{i} / \lambda_{0}$ in the fast and intermediate diffusion regime. The reaction barrier parameter is relatively high with (a) $\beta \Delta G^{*}=8$; (b) $\beta \Delta G *=9$; (c) $\beta \Delta G^{*}=10$. The dotted line indicates the plot for a function $\tau=\tau_{L} \exp \left(\beta \Delta G^{*}\right)$.
In the narrow reaction window limit, $\lambda_{i} / \lambda_{0}=0$, the dependence of $\tau_{a}$ on $\beta \Delta G *$ is given by Eq. (3.7a). For larger values of $\beta \Delta G^{*}$, Eq. (3.7a) can be approximated as ${ }^{40}$

$$
\tau_{a} \approx k_{e}^{-1}+\tau_{L} \int_{0}^{\sqrt{\overline{\Delta \Delta G^{*}}}} d y e^{y^{2}}
$$

The integral is related to Dawson's integral ${ }^{41}$ and approaches the value $\frac{1}{2}\left(\beta \Delta G^{*}\right)^{-1 / 2} \exp \left(\beta \Delta G^{*}\right)$ for large values of the free energy barrier parameter. Taking into account also the dependence of $k_{e}$ on $\beta \Delta G^{*}$, Eq. (2.9), we obtain the relation

$$
\tau_{a} \propto \exp \left(\beta \Delta G^{*}\right) \quad\left(\beta \Delta G^{*} \text { large }\right)
$$

for the dependence of the average survival time $\tau_{a}$ on $\beta \Delta G$ * in the narrow reaction window limit. With Eq. (2.10b) the relation

$$
\tau_{a} \propto \exp \left[\frac{1}{2} \beta \Delta G^{0}+\frac{1}{4}\left(\frac{\Delta G^{0}}{\lambda}\right) \beta \Delta G^{0}\right]
$$

follows for the dependence of $\tau_{a}$ on the experimentally controllable parameter $\beta \Delta G^{0}$. In particular, Eq. (3.10) predicts the usual linear dependence of $\ln \tau_{a}$ on $\beta \Delta G^{0}$ for small values of $\Delta G^{0} / \lambda$ with a slope of $\frac{1}{2}$.

Numerical results for the dependence of $\tau_{a}$ on $\beta \Delta G^{*}$ are shown in Fig. 8 for several values of the reaction window parameter $\lambda_{i} / \lambda_{0}$ and of the time-scale ratio $\tau_{L} k_{e}(0)$, where $k_{e}(0)$ is the value of $k_{e}$ at barrier height $\beta \Delta G^{*}=0$. Since $\tau_{b}$ is approximately equal to $\tau_{a}$ for larger values of $\beta \Delta G^{*}$ (see also the discussion of Fig. 7 above) we give only the results for $\tau_{a}$. We note that in Fig. 8 the time scale is normalized by $k_{e}(0)$, in contrast to the normalization of the time scale in Figs. 1 to 7. In Fig. 8(a) it is seen that in the narrow reaction window limit the relation (3.9) holds already for $\beta \Delta G^{*}>1$. Figures 8(b) and 8(c) show that this relation also holds for $\lambda_{i} / \lambda_{0} \neq 0$, although only ultimately for larger values of $\beta \Delta G^{*}$, depending on the magnitude of $\tau_{L} k_{e}(0)$. We note that in case of large values of $\tau_{L} k_{e}(0)$ the dependence of $\tau_{a}$ on $\beta \Delta G^{*}$ over some intermediate range of values for $\beta \Delta G^{*}$ can be approximated well by

$$
\tau_{a} \propto \exp \left(a \beta \Delta G^{*}\right),
$$

with $0<a<1$, instead of by Eq. (3.9).

The dependence of $\tau_{a}$ on $\beta \Delta G^{0}$ that follows from the results in Fig. 8 via the rescaling according to Eq. (2.10b) is shown in Fig. 9. We may note that, because of Eq. (2.10b), the actual dependence of $\tau_{a}$ on $\beta \Delta G^{\circ}$ depends also on the value of $\beta \lambda$. We have chosen a not atypical value of $\beta \lambda_{0}$ of 40 , corresponding to a value of about $24 \mathrm{kcal} / \mathrm{mol}$ for the solvent contribution $\lambda_{0}$ to the reorganization energy parameter $\lambda$ at room temperature. It is seen in Fig. 9 that even in the narrow reaction window limit, $\lambda_{i} / \lambda_{0}=0$, there is a quadratic dependence of $\ln \tau_{a}$ on $\beta \Delta G^{0}$ when $\beta \Delta G^{0}$ is varied over a large range. For small values of $\Delta G^{\circ} / \lambda$ a linear dependence with slope $\frac{1}{2}$ holds. Moreover, since for nonzero values of $\lambda_{i} / \lambda_{0}$ the behavior of $\tau_{a}$ deviates from the relation (3.9) over some range of $\beta \Delta G *$ for large $\tau_{L} k_{e}(0)$, there are also deviations from a quadratic dependence of $\ln \tau_{a}$ on $\beta \Delta G^{\circ}$ in these cases. This can also be seen from the fact that in Figs. 9(b) and 9(c) the curves for larger values of $\tau_{L} k_{e}(0)$ are no longer parallel to the curve for $\tau_{L} k_{e}(0)=1$, which still 


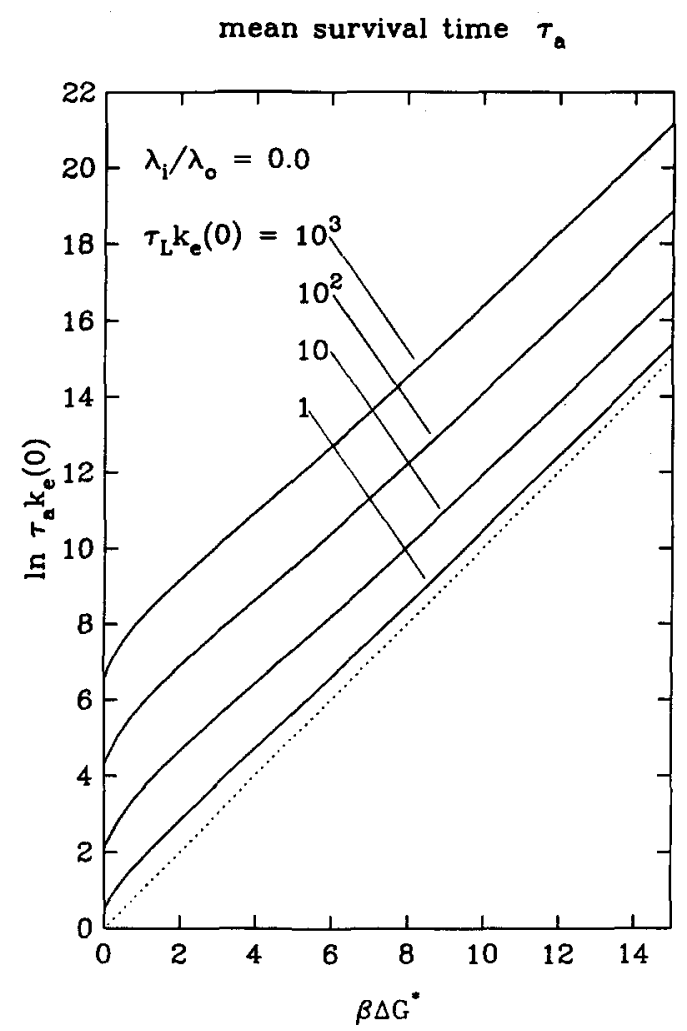

mean survival time $\tau_{a}$

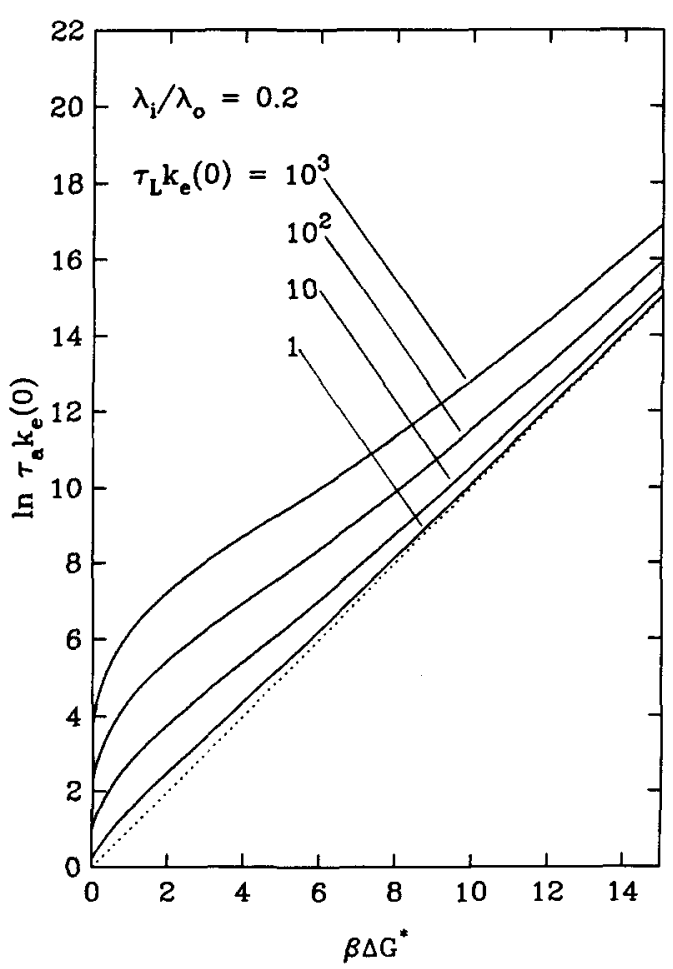

shows a quadratic dependence of $\ln \tau_{a}$ on $\beta \Delta G^{0}$.

In closing this section we like to summarize our results on the possibility of a simple approximate description of the results for $\tau_{a}$ and $\tau_{b}$. We have noted that Eq. (3.4) is a good approximation when $\lambda_{i} / \lambda_{0}=0$, and, in case of $\tau_{b}$, it is also

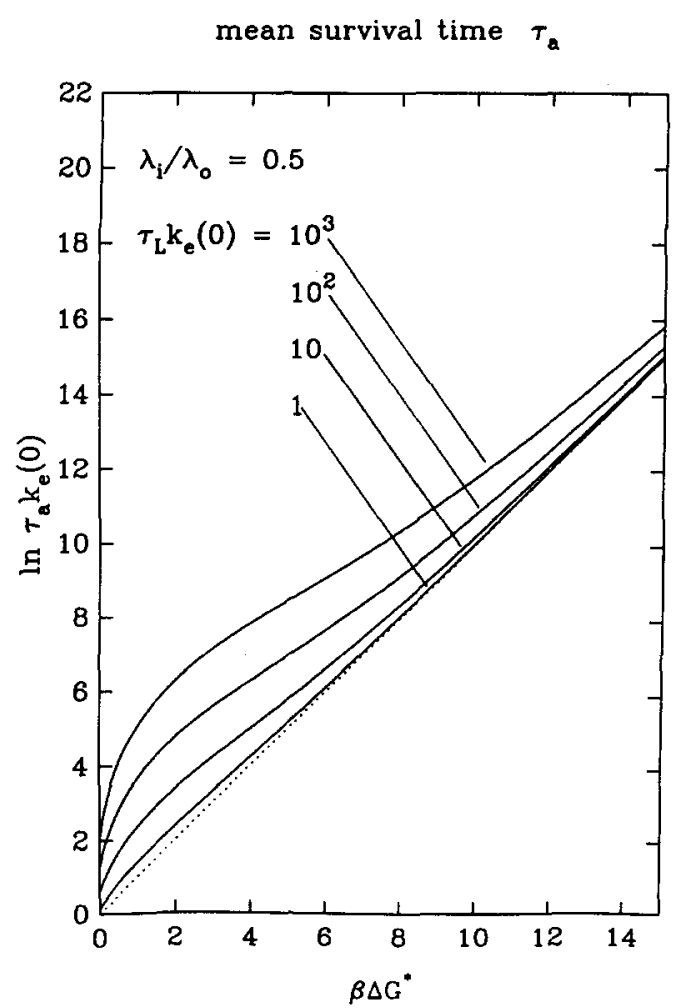

FIG. 8. Average survival time $\tau_{a}$ vs reaction barrier parameter $\beta \Delta G *$ for various values of the reaction window parameter $\lambda_{i} / \lambda_{0}$ and of the time-scale ratio $\tau_{L} k_{e}(0)$; (a) $\lambda_{i} / \lambda_{0}=0$; (b) $\lambda_{i} / \lambda_{0}=0.2$; (c) $\lambda_{i} / \lambda_{0}=0.5$. The time scales are normalized to $k_{e}\left(\beta \Delta G^{*}=0\right)$, in contrast to the normalization in Figs. 1 to 7. 
mean survival time $\tau_{\text {a }}$

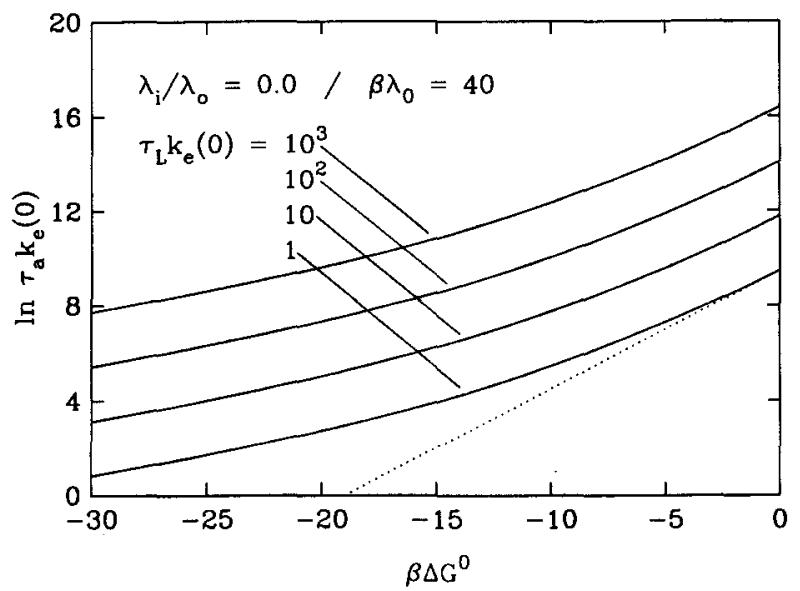

mean survival time $\tau_{a}$

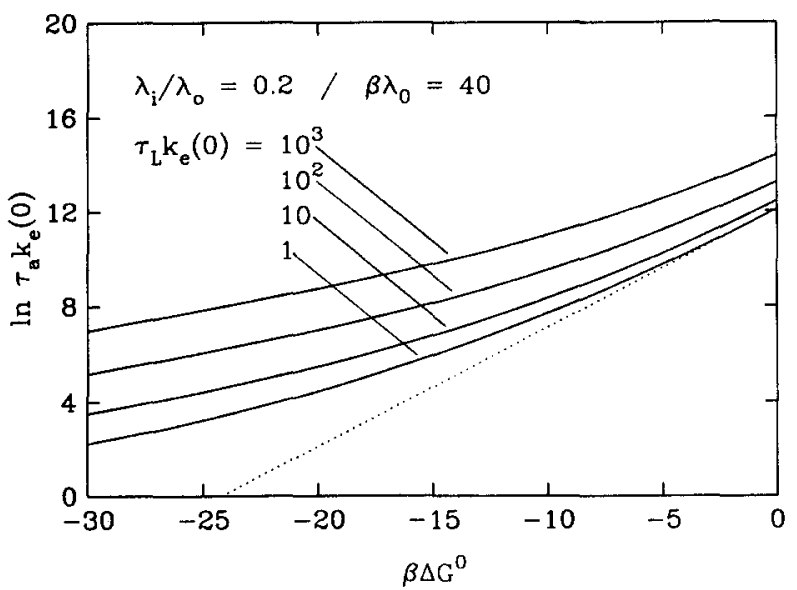

mean survival time $\tau_{\mathbf{a}}$

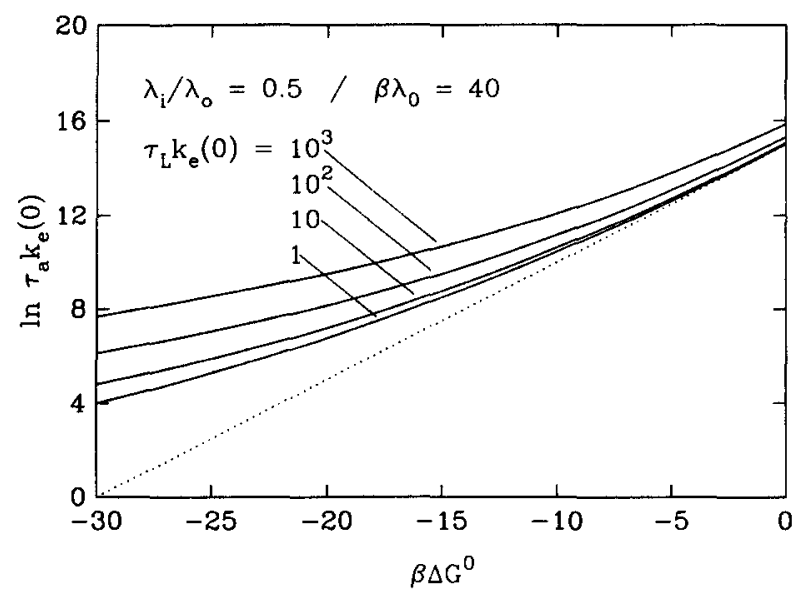

FIG. 9. Average survival time $\tau_{a}$ vs reaction standard free energy $\beta \Delta G^{0}$ for various values of the reaction window parameter $\lambda_{i} / \lambda_{0}$ and of the time-scale ratio $\tau_{L} k_{e}(0)$; (a) $\lambda_{i} / \lambda_{0}=0$; (b) $\lambda_{i} / \lambda_{0}=0.2$; (c) $\lambda_{i} / \lambda_{0}=0.5$. The time scales are normalized to $k_{e}\left(\beta \Delta G^{*}=0\right)$, in contrast to the normalization in Figs. 1 to 7 . The dotted lines denote the relation $\tau_{a} \propto \exp \left(\frac{1}{2} \beta \Delta G^{0}\right)$ which holds for small values of $\Delta G^{\circ} / \lambda$. exponential. In that case the relaxation time $\tau_{a}$ becomes equal to the inverse of the lowest eigenvalue of the operator $k(X)-\mathbf{L}^{(s)}(X)$. An alternative and more familiar way to calculate $\tau_{a}$ would be in this case to use standard methods for solving one-dimensional quantum mechanical eigenvalue problems, instead of solving Eqs. (2.35) or $\left(2.35^{\prime}\right)$, respectively, for the auxiliary function $\mu_{-1}(X)$ and using Eq. (2.33) to calculate the generalized moment $\mu_{-1}$, which is equal to $\tau_{a}$.

\section{RESULTS ON THE RELAXATION OF $\boldsymbol{Q}(\boldsymbol{t})$}

We have also investigated the relaxational behavior of $Q(t)$ by employing the generalized moment algorithm of Sec. II B. The approximations $q(t)$ to $Q(t)$ presented below were $N$-exponential approximants (2.23) with the order $N$ chosen so that approximants of higher order did not give rise to a recognizable change in the plotted behavior. This choice can be regarded as sufficiently exact since, in our experience, the convergence rate of the approximation algorithm of Sec. II B is quite fast, in general. The order $N$ of these numerical solutions varied between 3 and 10 in the various cases, corresponding to the degree of multiexponentiality $Q(t)$ exhibited. In the fast diffusion regime $\left(\tau_{L} k_{e}<1\right)$ the relaxation of $Q(t)$ is in effect single exponential and we refrain from giving examples for this case.

In Fig. 10 an example is given for the behavior of $Q(t)$ in the intermediate $\tau_{L}$ regime, i.e., for $\tau_{L} k_{e} \approx 1$, with $\lambda_{i} / \lambda_{0}$ $=0.2$. As can be seen, the relaxation is still almost single exponential. The approximations $q_{a}(t)$ and $q_{b}(t)$, Eqs. (2.26) and (2.28), respectively, describe the relaxation equally well, with $q_{b}(t)$ becoming exact in the long time limit. The bi-exponential description $q_{2}(t)$, Eq. (2.27), is indistinguishable from the numerical solution.

surviving fraction $Q(t)$

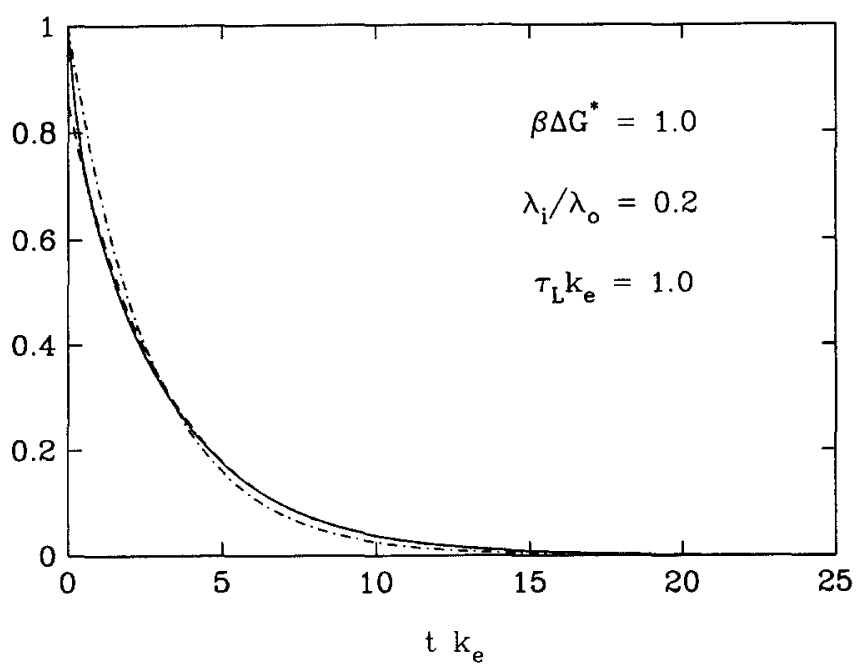

FIG. 10. Time behavior of the survival probability $Q(t)$ in the intermediate diffusion regime, $\tau_{L} k_{e}=1$. Reaction parameters are $\lambda_{i} / \lambda_{0}=0.2$ and $\beta \Delta G^{*}=1$. ( $(-)$ is the numerical solution (see the text); $\left(-^{\cdot-\cdot}\right)$ is the single-exponential approximation $q_{a}(t)$, Eq. $(2.25) ;(--)$ is the long-time approximation $q_{b}(t), \mathrm{Eq} .(2.27) ;(\cdots)$ is the bi-exponential approximation $q_{2}(t)$, Eq. (2.26). The function $q_{2}(t)$ is indistinguishable from the numerical solution in this case. 
surviving fraction $Q(t)$

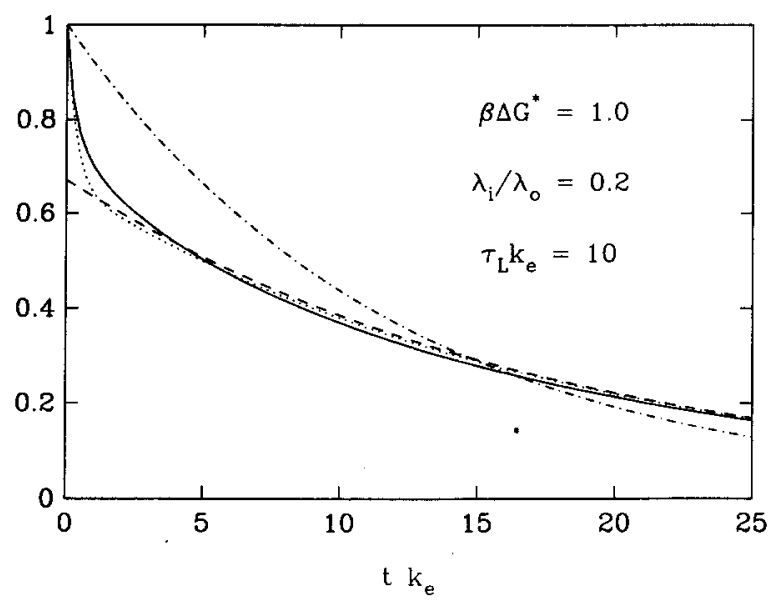

FIG. 11. Same as Fig. 10 for $\tau_{L} k_{e}=10$.

For still larger values of $\tau_{L} k_{e}$ at this value of $\lambda_{i} / \lambda_{0}$, the quality of single- and also of bi-exponential approximations become less acceptable. Figure 11 gives an example for the value $\tau_{L} k_{e}=10$. In this case the single-exponential approximations already give an incorrect description of the relaxation process of $Q(t)$, although $q_{b}(t)$ captures the asymptotic long-time behavior and can still be considered an acceptable approximation for this time regime. A bi-exponential description is still a good approximation in this intermediate diffusion case, as demonstrated by $q_{2}(t)$ in Fig. 11. Figure 12 gives an example for an already somewhat extreme case of the time-scale ratio, i.e., for $\tau_{L} k_{e}=10^{3} . Q(t)$ exhibits a truly multiexponential relaxation behavior and an approximation of order $N=10$ constitutes the numerical solution in this case. The single- and bi-exponential approximations give now a totally wrong impression of the true behavior of $Q(t)$. For example, $q_{2}(t)$ indicates an equilibration process to a quasistationary state which certainly does not take place. Rather, an algebraic decay

$$
Q(t) \propto t^{-r}
$$

occurs over a range of about two to three decades, namely in

surviving fraction $Q(t)$

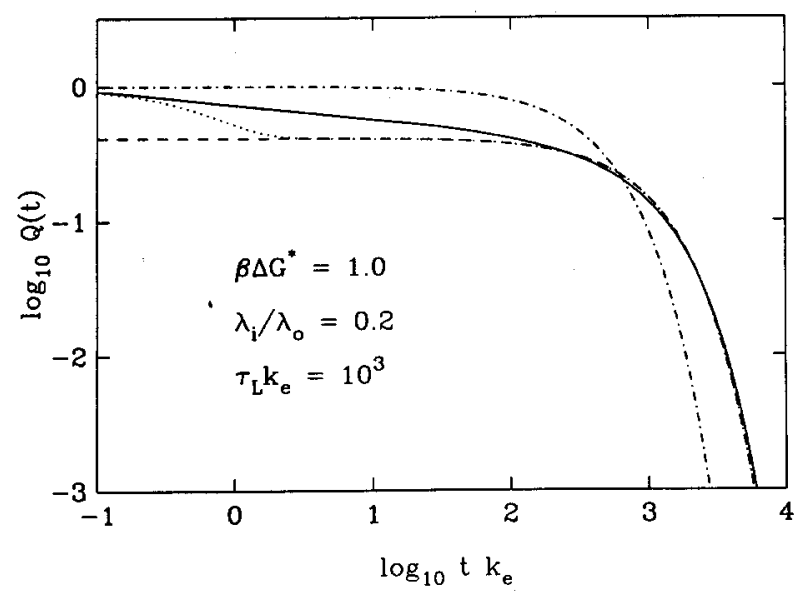

FIG. 12. Same as Fig. 10 for $\tau_{L} k_{e}=10^{3}$. surviving fraction $Q(t)$

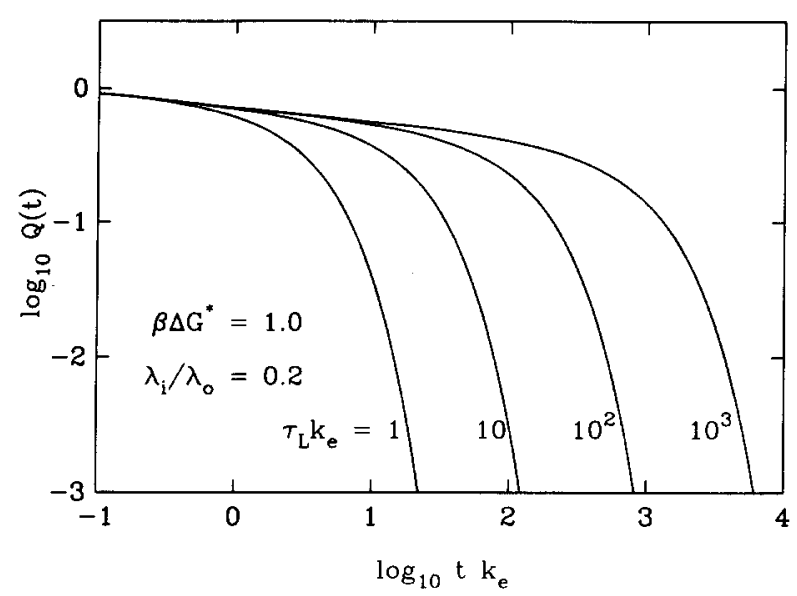

FIG. 13. Time behavior of the survival probability $Q(t)$ for different values of the time-scale ratio $\tau_{L} k_{e}$. Reaction parameters are $\lambda_{i} / \lambda_{0}=0.2$ and $\beta \Delta G^{*}=1$. There is seen to be an almost algebraic decay, Eq. (4.1), with $\gamma \approx 0.1$ in the time range $10^{-1}<t k_{e}<10^{2}$ for $\tau_{L} k_{e}=10^{3}$.

the range $10^{-1}<t k_{e}<10^{2}$. This algebraic decay goes over, in the long-time limit, to an exponential relaxation described by $q_{b}(t)$. In the example of Fig. 12 the exponent $\gamma$ has a value of about 0.1 . In general, the exponent $\gamma$ depends on the reaction parameters of the system.

Figures 13 to 16 are intended to give an overview of the relaxational behavior of $Q(t)$ for different values of the reaction window parameter $\lambda_{i} / \lambda_{0}$, the free energy barrier parameter $\beta \Delta G^{*}$, and the time-scale ratio $\tau_{L} k_{e}$.A common feature in the different curves is the algebraic character of the relaxation, Eq. (4.1), over some range of time for large values of the time-scale ratio $\tau_{L} k_{e}$ as seen in the figures and discussed in the legends to the figures. The algebraic character of the relaxation is particularly noticeable for smaller, but nonzero, values of both the reaction window parameter and of the free energy barrier parameter. For large values of these parameters the curves tend to approach a single-exponential

surviving fraction $Q(t)$

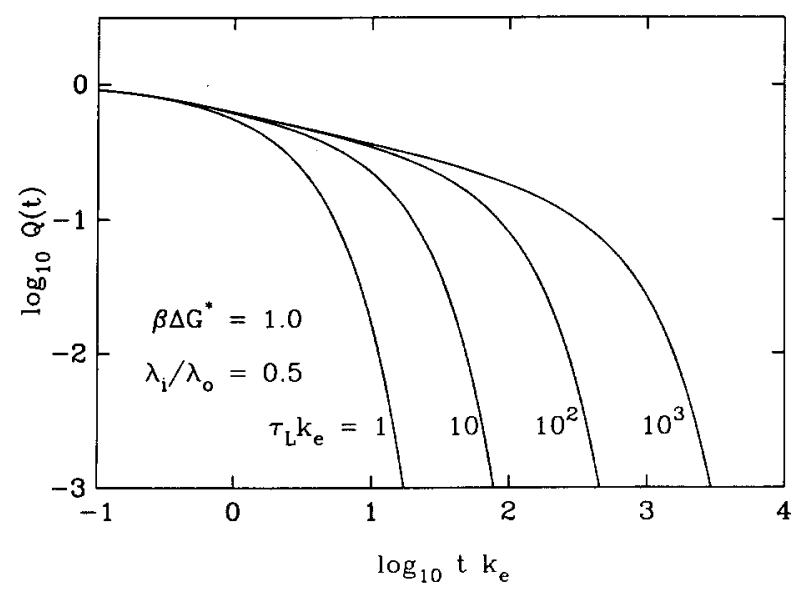

FIG. 14. Same as Fig. 13 with $\lambda_{i} / \lambda_{0}=0.5$. There is seen to be an almost algebraic decay, Eq. (4.1), with $\gamma \approx 0.2$ in the time range $10^{-0.5}<t k_{e}<10^{1.5}$ for $\tau_{L} k_{e}=10^{3}$. 
surviving fraction $Q(t)$

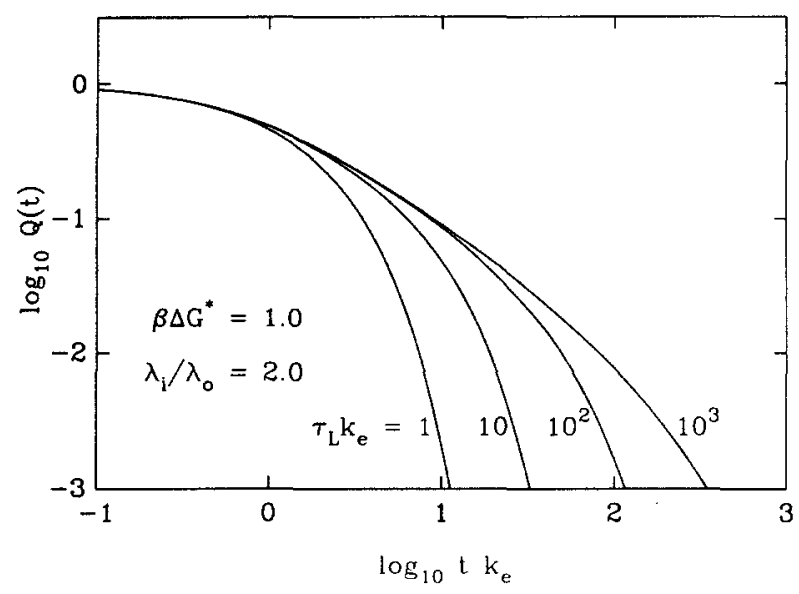

FIG. 15. Same as Fig. 13 with $\lambda_{i} / \lambda_{0}=2.0$.

behavior, as was already discussed for the limiting cases in Sec. II A and in Sec. III.

In closing this section we would like to comment on the behavior of $Q(t)$ in the nondiffusing limit $\tau_{L} k_{e} \rightarrow \infty$. In this case the application of the generalized moment algorithm is not possible since the low-frequency moments diverge, the divergence of each depending on $\lambda_{i} / \lambda_{0}$ [see, for example, Eqs. (3.1) for $\mu_{-1}$ and $\mu_{-2}$ ]. Therefore, a determination of the time behavior of $Q(t)$ has to rely on a numerical evaluation of the integral (2.14). However, since in this limiting case no solvent dynamical effects influence the electron transfer process we refrain from giving examples. Moreover, the case of a distribution of reaction rates has previously been frequently treated, e.g., as a distribution of distances between donors and acceptors in electron transfer reactions $\mathrm{s}^{42}$ or as a distribution of conformational substates in the low-temperature reaction dynamics of proteins. ${ }^{43}$

surviving fraction $Q(t)$

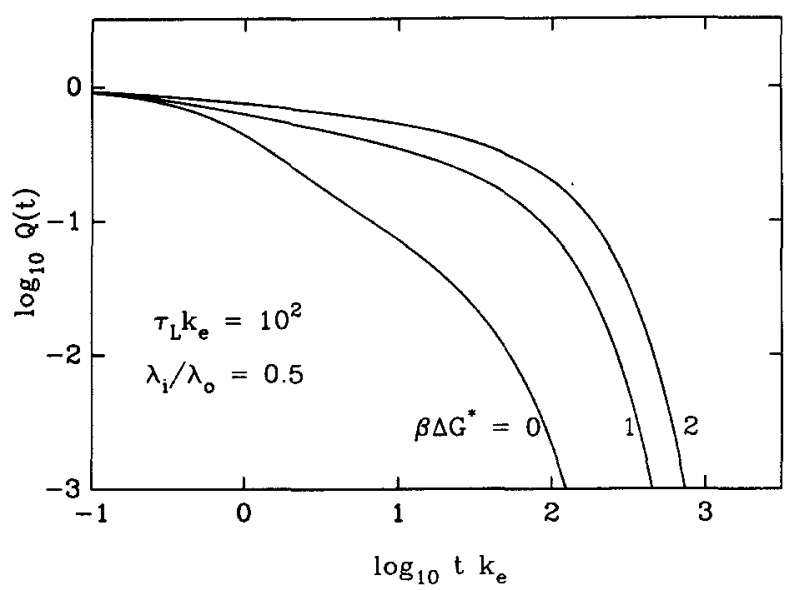

FIG. 16. Time behavior of the survival probability $Q(t)$ for different values of the reaction barrier parameter $\beta \Delta G *$. Parameters $\lambda_{i} / \lambda_{0}=0.5$ and $\tau_{L} k_{e}$ $=10^{2}$. There is seen to be an almost algebraic decay, Eq. (4.1), with $\gamma \approx 0.8$ in the time range $10^{\circ} \leqslant t k_{e} \leqslant 10^{1}$ for $\beta \Delta G^{*}=0$.

\section{DISCUSSION}

We first comment on the methodological approach. The results presented in the last two sections demonstrate that the generalized moment algorithm of Secs. II B and II C is a rather powerful method for the analysis of reaction-diffusion equations over the whole range of parameters. In particular, even the partly algebraic relaxation, Eq. (4.1), of $Q(t)$ in the slow diffusion regime could be revealed by this method, although the functional form of the approximation is multiexponential only. In the fast and intermediate diffusion regimes the simplified single- and bi-exponential approximations (2.25) and (2.26) were seen to be quite accurate descriptions of $Q(t)$. The single-exponential approximation (2.27) based on $\tau_{b}$ gave the correct asymptotic long-time behavior in any event, even in the slow diffusion regime.

Results on the survival probability $Q(t)$ are of particular interest in that they point to conditions $\left(\tau_{L} k_{e}\right.$ small or $\beta \Delta G^{*}$ large) for observing largely single-exponential behavior and to other conditions $\left(\tau_{L} k_{e}\right.$ large, $\beta \Delta G *$ small and intermediate values of $\lambda_{i} / \lambda_{0}$ ) for observing multiexponential behavior. Experiments which focus on solvent dynamical effects in the electron transfer process are comparatively new and little attention has been devoted thus far to the experimental distinction between the two types of behavior of $Q(t)$.

Some experiments made several years ago touch on this question of single- vs multiexponential behavior. Two different experimental groups studied the same system, the intramolecular electron transfer of dimethylaminobenzonitrile in 1-propanol.,3 One group reported single-exponential decay and the other a double-exponential decay. Using improved short light pulse techniques it should be possible to determine which behavior is correct.

One interesting result of the present paper is the approximate power-law dependence of $\tau_{a}$, i.e., of the estimate for the experimentally determined inverse electron transfer rate, on the solvent relaxation time $\tau_{L}$ in the large $\tau_{L}$ regime. This behavior is in strong contrast to the results of other models that describe the influence of solvent polarization fluctuations on electron transfer reactions for solvents with a single polarization relaxation time $\tau_{L} \cdot{ }^{12-15,17}$ From these models only a simple linear dependence $\tau \propto \tau_{L}$ is derived for the inverse electron transfer rate. The existence of the fractional exponent $\alpha$ in Eq. (3.2) in a single relaxation time solvent is due to the inclusion in the present model of the contributions from intramolecular coordinates to the electron transfer process.

Kosower and Huppert ${ }^{1}$ studied experimentally an intramolecular electron transfer in a series of alcoholic solvents. For the solvents for which actual reaction times were determined, propanol through decanol, their $\tau_{L}$ 's varied from 10 to $460 \mathrm{ps}$. Their $k_{e}$ appears to be larger than $0.1 \mathrm{ps}^{-1}$, the reciprocal of the pulse-limited rise time for the fastest system studied (methanol as solvent), and so $\tau_{L} k_{e}$ was varied from more than unity to more than about 50 . The reaction time was reported to equal $\tau_{L}$, within the experimental error for those systems. From Figs. 1 and 2 we infer that for their systems $\lambda_{i} / \lambda_{0}$ and $\beta \Delta G^{*}$ are both approximately zero. It would be especially desirable, however, to see if any depar- 
ture from this equality or from single-exponential behavior can be found (apart from that due to the existence of several dielectric relaxation times in the solvent) using solutes with some nonnegligible value of $\lambda_{i} / \lambda_{0}$. Deviations from $\tau \approx \tau_{L}$ would then be expected, even when $\beta \Delta G^{*} \approx 0$. We have discussed elsewhere some other relevant considerations, namely which $\tau_{L}$, "constant charge" dielectric relaxation time, to use actually ${ }^{19(a)}: \tau_{L}$ can be written as $\left(\epsilon_{o} / \epsilon_{s}\right) \tau_{D}$, where $\tau_{D}$ is the customary constant electric field relaxation time and $\epsilon_{o}$ and $\epsilon_{s}$ are the relevant high-frequency ("optical") and lowfrequency ("static") dielectric constants of the solvent, respectively. ${ }^{44}$ The reader is referred to Ref. 19 (a) for a discussion of which dielectric constants are relevant.

A very slow relaxation of the solvent polarization occurs near a glass transition of the solvent. McGuire and McLendon $^{9}$ recently investigated electron transfer processes in such a situation. These authors studied the electron transfer reaction between methyl viologen $\left(\mathrm{MV}^{2+}\right)$ and electronically excited ruthenium complexes such as $\mathrm{Ru}(\mathrm{phen})_{3}^{2+}$, with rigid glycerol as solvent. They reported a power-law dependence of the electron transfer rate on the solvent polarization relaxation time $\tau_{L}$ in their system, valid over several decades of the solvent relaxation time. The exponent was about 0.6 . Such a result, on the surface at least, may indicate that $\tau_{L} k_{e}$ is in an intermediate range (e.g., the slope of the $\tau_{a} k_{e}$ vs $\tau_{L} k_{e}$ plot in Fig. 1 for $\lambda_{i} / \lambda_{0}=0$ and $\beta \Delta G^{*}=0$ is less than unity when $\tau_{L} k_{e}$ is in an intermediate range), or that there is a strong contribution from intramolecular coordinates to the electron transfer process. However, the analysis of the experiment is somewhat indirect, since the reaction rates were inferred from the quenching data using a Perrin model, and then were recalculated to rates at a fixed separation distance (15 $\AA$ ) using an exponential model. The $\tau_{L}$ was varied from about $10^{-8} \mathrm{~s}$ to more than $10^{-1} \mathrm{~s}$ by varying the temperature. ${ }^{45}$ The reaction time itself was estimated, indirectly, to vary by somewhat less than three orders of magnitude. The $k_{e}$ at these temperatures was estimated ${ }^{46}$ to vary only from $10^{6} \mathrm{~s}^{-1}$ to $10^{5} \mathrm{~s}^{-1}$ over the same temperature range, giving a variation of $\tau_{L} k_{e}$ from $10^{-2}$ to $10^{4}$. Clearly it would be useful to investigate other systems, preferably intramolecular electron transfer systems with a fixed geometry, and so avoid some of the uncertainties present in this especially interesting first study. A direct determination of the functional form of $Q(t)$ for this case, an important question, would then also be possible.

\section{ACKNOWLEDGMENTS}

We gratefully acknowledge the support of this research by an Office of Naval Research contract and a grant from the National Science Foundation. W. N. is pleased also to acknowledge support by a grant from the Deutsche Forschungsgemeinschaft (Schu 523/1-1) during a period where some of the concepts used in this paper were developed.

\section{APPENDIX A: LOW-FREQUENCY MOMENTS IN THE NARROW REACTION WINDOW LIMIT}

As we have noted in Sec. II, in the narrow reaction window limit, $\lambda_{i} / \lambda_{0} \rightarrow 0$, the reactive term Eq. (2.6') assumes the form of a delta function

$$
k(X)=k_{e}\left[P_{0}\left(X_{c}\right)\right]^{-1} \delta\left(X-X_{c}\right) .
$$

Because of this simple form integral expressions for the lowfrequency moments can be derived. In contrast to the numerical evaluation of the moments in Sec. II C, the analytical derivation of these expressions is simpler by employing the adjoint Fokker-Planck operator of Sec. II B. The differential equation for the adjoint auxiliary functions is then ${ }^{21}$ $(n>0)$

$$
\left[k(X)-\mathbf{L}^{+}(x)\right] \mu_{-n}^{+}(X)=\mu_{-(n-1)}^{+}(X),
$$

with $\mu_{0}^{+}(X)=1$ and the boundary conditions $(d / d X)$ $\times\left.\mu_{-n}^{+}(X)\right|_{X= \pm \infty}=0$. It can be solved in a straightforward way, e.g., as in Ref. 47, with the result

$$
\begin{aligned}
\mu_{-n}^{+}(X)= & k_{e}^{-1}+\tau_{L} H\left(X-X_{c}\right) \int_{X_{c}}^{X} d Y\left[P_{0}(Y)\right]^{-1} \\
& \times \int_{Y}^{\infty} d Z P_{0}(Z) \mu_{-(n-1)}^{+}(Z) \\
& +\tau_{L} H\left(X_{c}-X\right) \int_{X}^{X_{c}} d Y\left[P_{0}(Y)\right]^{-1} \\
& \times \int_{-\infty}^{Y} d Z P_{0}(Z) \mu_{-(n-1)}^{+}(Z)
\end{aligned}
$$

In this formula $H(X)$ denotes the Heaviside step function: $H(X)=0$ for $X<0$ and $H(X)=1$ for $X>0$, and its derivative $H^{\prime}(X)$ is $\delta(X)$. With $P_{0}(X)$ being the Boltzmann distribution (2.4) for a general potential, Eq. (A3) holds also for potentials more complicated than the harmonic one considered in our paper. From the auxiliary function Eq. (A3) integral expressions for the first two low-frequency moments can be derived using the relations

$$
\begin{aligned}
& \mu_{-1}=\left\langle 1 \mid \mu_{-1}^{+}(X)\right\rangle_{0}=\left\langle\mu_{-1}^{+}(X)\right\rangle_{0}, \\
& \mu_{-2}=\left\langle 1 \mid \mu_{-2}^{+}(X)\right\rangle_{0}=\left\langle\mu_{-1}^{+}(X) \mid \mu_{-1}^{+}(X)\right\rangle_{0},
\end{aligned}
$$

with the results

$$
\begin{aligned}
& \mu_{-1}=k_{e}^{-1}+\tau_{L} I_{1}, \\
& \mu_{-2}=k_{e}^{-2}+2 k_{e}^{-1} \tau_{L} I_{1}+\tau_{L}^{2} I_{2},
\end{aligned}
$$

where we have used the abbreviations for the integrals

$$
\begin{aligned}
I_{1}\left(X_{c}\right)= & \int_{-\infty}^{X_{c}} d X\left[P_{0}(X)\right]^{-1}\left[\int_{-\infty}^{X} d Y P_{0}(Y)\right]^{2} \\
& +\int_{X_{c}}^{\infty} d X\left[P_{0}(X)\right]^{-1}\left[\int_{X}^{\infty} d Y P_{0}(Y)\right]^{2}, \\
I_{2}\left(X_{c}\right)= & \int_{-\infty}^{X_{c}} d X P_{0}(X)\left[\int_{X}^{X_{c}} d Y\left[P_{0}(Y)\right]^{-1}\right. \\
& \left.\times \int_{-\infty}^{Y} d Z P_{0}(Z)\right]^{2} \\
& +\int_{X_{c}}^{\infty} d X P_{0}(X)\left[\int_{X_{c}}^{X} d Y\left[P_{0}(Y)\right]^{-1}\right. \\
& \left.\times \int_{Y}^{\infty} d Z P_{0}(Z)\right]^{2}
\end{aligned}
$$

These integrals can be evaluated analytically for functional forms of $P_{0}(X)$ such as polynomials or simple exponentials. In our case the Boltzmann distribution is, instead, a Gaussian and we have used a numerical quadrature for the evalua- 
TABLE IV. Numerical values for the functions $I_{1}\left(X_{c}\right)$ and $I_{2}\left(X_{c}\right)$, Eq. (A8a).

\begin{tabular}{rllll}
\hline$\beta \Delta G^{*}$ & $X_{c}$ & $I_{1}\left(X_{c}\right)$ & $I_{2}\left(X_{c}\right)$ & $I_{2}\left(X_{c}\right) / I_{1}\left(X_{c}\right)$ \\
\hline 0 & 0.000 & 0.6931 & 0.6515 & 0.9400 \\
1 & 1.414 & 3.584 & $1.513(1)$ & 4.222 \\
2 & 2.000 & 9.696 & $9.953(1)$ & 9.984 \\
3 & 2.449 & $2.342(1)$ & $5.591(2)$ & $2.387(1)$ \\
4 & 2.828 & $5.556(1)$ & $3.107(3)$ & $5.592(1)$ \\
5 & 3.162 & $1.331(2)$ & $1.777(4)$ & $1.335(2)$ \\
6 & 3.464 & $3.245(2)$ & $1.054(5)$ & $3.248(2)$ \\
7 & 3.742 & $8.036(2)$ & $6.459(5)$ & $8.037(2)$ \\
8 & 4.000 & $2.017(3)$ & $4.069(6)$ & $2.017(3)$ \\
9 & 4.243 & $5.117(3)$ & $2.619(7)$ & $5.117(3)$ \\
10 & 4.472 & $1.309(4)$ & $1.714(8)$ & $1.309(4)$ \\
\hline
\end{tabular}

${ }^{a}$ Numbers in parentheses indicate powers of 10.

tion of Eq. (A6). However, an analytical simplification of the multiple integrals in Eq. (A6) is still obtainable which simplifies also the numerical quadrature: For $X_{c}=0$ and $k_{e}$ $\rightarrow \infty$ it has been shown in Part I that the analytical solution for the survival probability is $Q(t)=(2 / \pi) \sin ^{-1}\left(e^{-t / \tau_{L}}\right)$. Since the moments are alternatively given by time integrals over $Q(t)$, as in Eq. (2.24), from the quoted result the value of the integrals at $X_{c}=0$ can be shown to be $e^{18,48}$

$$
\begin{aligned}
& I_{1}(0)=\ln 2, \\
& I_{2}(0)=\frac{\pi^{2}}{24}+\frac{1}{2}(\ln 2)^{2} .
\end{aligned}
$$

After some algebra, the corrections due to a nonzero value of $X_{c}$ can be put into the form

$$
\begin{aligned}
I_{1}\left(X_{c}\right)= & I_{1}(0)+2 \sqrt{\pi} F_{1}\left(\sqrt{\beta \Delta G^{*}}\right) \\
I_{2}\left(X_{c}\right)= & I_{2}(0)+2 \sqrt{\pi}(\ln 2) F_{1}\left(\sqrt{\beta \Delta G^{*}}\right) \\
& +2 \pi\left[F_{1}\left(\sqrt{\beta \Delta G^{*}}\right)\right]^{2} \\
& +\pi\left[D\left(\sqrt{\beta \Delta G^{*}}\right)\right]^{2}+2 \pi F_{2}\left(\sqrt{\beta \Delta G^{*}}\right),
\end{aligned}
$$

where we have used the relation between $X_{c}$ and $\beta \Delta G^{*}$, Eq. (2.10'). $D(x)=\int_{0}^{x} d z e^{z^{z}}$ is related to Dawson's integral, which is tabulated in Ref. 41, and the integrals $F_{1}(x)$ and $F_{2}(x)$ are given by

$$
\begin{aligned}
& F_{1}(x)=\int_{0}^{x} d y e^{y^{2}} \operatorname{erf}(y), \\
& F_{2}(x)=\int_{0}^{x} d y e^{y^{2}} \int_{0}^{y} d z e^{z^{2}}[\operatorname{erf}(z)]^{2},
\end{aligned}
$$

with

$$
\operatorname{erf}(z)=\frac{2}{\sqrt{\pi}} \int_{0}^{z} d t e^{-t^{2}}
$$

being the error function. The integrals $F_{1}(x)$ and $F_{2}(x)$ can be evaluated by standard numerical methods ${ }^{35}$ and numerical values for $I_{1}\left(X_{c}\right)$ and $I_{2}\left(X_{c}\right)$ for different values of the parameter $\beta \Delta G^{*}$ of our model are given in Table IV.

In closing this Appendix we note that the narrow reaction window limit of the linear $\tau_{L}$ approximation for the average survival time $\tau_{\alpha}$ (and, therefore, for the first low- frequency moment $\mu_{-1}$ presented in Sec. III), Eqs. (3.3a) and (3.4) with $c=0$ is identical to the expression Eq. (A5a) for $\mu_{-1}$. This identity can be easily proven by comparing the derivatives of (3.3a) and of (A5a) with respect to the parameter $\beta \Delta G^{*}$ and taking into account the representation (A9a) for $I_{1}\left(X_{c}\right)$. These derivatives are identical and so are the values of the functions at $X_{c}=0$, which finishes the proof.

Therefore, in the narrow reaction window limit the first order approximation (3.3a) for the first low-frequency moment is already exact. However, a similar property does not hold for the second moment, since Eq. (A5b) shows that it contains a term of second order in $\tau_{L}$.

\section{APPENDIX B: NONSINGULARITY OF THE MATRIX ELEMENT IN EQ. (3.3)}

We may begin this discussion by noting that any function $f(X)$ can be expanded in eigenfunctions $\psi_{n}^{+}(X)$ of the adjoint Fokker-Planck operator $\mathrm{L}^{+}(X)$,

$$
f(X)=\sum_{n=0}^{\infty} f_{n}^{+} \psi_{n}^{+}(X)
$$

Since the operators $L(X)$ and $L^{+}(X)$ are non-Hermitian but are adjoint to each other, their respective eigenvalues are equal and their eigenfunctions, $\psi_{n}(X)$ and $\psi_{n}^{+}(X)$, respectively, can be normalized so that the "quasi-orthogonality" relation

$$
\int_{-\infty}^{\infty} d X \psi_{n}(X) \psi_{m}^{+}(X)=\delta_{n m}
$$

holds. ${ }^{23}$ Therefore, the expansion coefficients $f_{n}^{+}$in Eq. (B1) can be written in terms of the projection of $f(X)$ on the eigenfunctions of $\mathrm{L}(X)$, i.e.,

$$
f_{n}^{+}=\int_{-\infty}^{\infty} d X \psi_{n}(X) f(X)
$$

Using Eq. (B1), the application of any integer power of the operator $\mathrm{L}^{+}(X)$ on the function $f(X)$ can now be represented as

$$
\left[\mathbf{L}^{+}(X)\right]^{v} f(X)=\sum_{n=0}^{\infty} \lambda_{n}^{v} f_{n}^{+} \psi_{n}^{+}(X),
$$

where $\lambda_{n}$ is the eigenvalue of $L(X)$ and $\mathbf{L}^{+}(X)$ correspond- 
ing to the $n$th eigenfunction. However, since $L(X)$ and $\mathrm{L}^{+}(X)$ have a single zero eigenvalue $\lambda_{0}=0, \mathrm{Eq} .(\mathrm{B} 4)$ is not well defined in case of $v<0$, in general, due to the singularity of the term corresponding to $n=0$.

On the other hand, any function $f(X)$ with a zero expansion coefficient $f_{0}^{+}$, i.e., any function that lies outside the null space of $\mathbf{L}^{+}(X)$, will lead to an expression (B4) that is well defined also for $v<0$. Using the projection operator $\mathbf{P}_{0}^{+}$, defined through

$$
\begin{aligned}
\mathbf{P}_{0}^{+} f(X) & =\psi_{0}^{+}(X) \int_{-\infty}^{\infty} d X^{\prime} \psi_{0}\left(X^{\prime}\right) f\left(X^{\prime}\right) \\
& =\psi_{0}^{+}(X) f_{0}^{+},
\end{aligned}
$$

which projects onto the one-dimensional null space of $\mathbf{L}^{+}(X)$, the property of to lie outside the null space can be stated for a function $f(X)$ as

$$
\left[1-\mathbf{P}_{0}^{+}\right] f(X)=f(X) \text {. }
$$

Since the eigenfunctions of $\mathrm{L}(X)$ and $\mathrm{L}^{+}(X)$ corresponding to $\lambda_{0}=0$ are the thermal equilibrium distribution and the unity function, ${ }^{23}$ respectively, i.e.,

$$
\psi_{0}(X)=P_{0}(X)
$$

and

$$
\psi_{0}^{+}(X)=1,
$$

it can be seen easily that the function

$$
\delta k(X)=k(X)-k_{0}{ }^{+} \psi_{0}{ }^{+}(X)
$$

fulfills the property (B6). Since $k_{0}^{+}$equals $k_{e}$, compare Eq. (B3), this function has the same form as in the main text, $\delta k(X)=k(X)-k_{\mathrm{e}}$. Therefore, the quantity $\left[\mathbf{L}^{+}(X)\right]^{\nu} \delta k(X)$ can be expressed in terms of eigenfunctions $\psi_{n}^{+}(X)$ according to Eq. (B4) in a well-defined manner even for $v<0$. From this it follows that the matrix element of Eq. (3.3) is also well defined.

We may note that in Refs. 20 and 49 it was demonstrated how to actually calculate such matrix elements as in Eq. (3.3). For general one-dimensional Fokker-Planck operators it was shown in Ref. 49 by employing the projection operator (B5) and property (B6) that matrix elements like in Eq. (3.3) can be expressed analytically in terms of multiple integrals, i.e., in our case

$$
\begin{aligned}
& \left\langle 1\left|\delta k(X)\left[\mathrm{L}^{+}(X)\right]^{-1} \delta k(X)\right| 1\right\rangle_{0} \\
& \quad=\tau_{L} \int_{-\infty}^{\infty} d X\left[P_{0}(X)\right]^{-1}\left[\int_{-\infty}^{X} d Y P_{0}(Y) \delta k(Y)\right]^{2} .
\end{aligned}
$$

This provides another way to derive Eq. (3.5), alternative to the one used in part $I$.

${ }^{1}$ E. M. Kosower and D. Huppert, Chem. Phys. Lett. 96, 433 (1983); D. Huppert, H. Kassety, and E. M. Kosower, Faraday Discuss. Chem. Soc. 74, 199 (1982).

${ }^{2}$ Y. Wang, M. McAuliffe, F. Novak, and K. B. Eisenthal, J. Phys. Chem. 85, 3736 (1981).
${ }^{3}$ D. Huppert, S. D. Rand, P. M. Rentzepis, P. F. Barbara, W. S. Struve, and Z. R. Grabowski, J. Chem. Phys. 75, 5714 (1981).

${ }^{4} M$. J. Weaver and T. Gennet, Chem. Phys. Lett. 113, 213 (1985).

${ }^{5}$ T. Gennet, D. F. Milner, and M. J. Weaver, J. Phys. Chem. 89, 2787 (1985).

${ }^{6}$ W. Harrer, G. Gramp, and W. Jaenicke, Chem. Phys. Lett. 112, 263 (1984).

${ }^{7}$ J. T. Hupp and M. J. Weaver, J. Phys. Chem. 89, 1601 (1985).

${ }^{8}$ X. Zhang, J. Leddy, and A. J. Bard, J. Am. Chem. Soc. 107, 3719 (1985).

${ }^{9}$ M. McGuire and G. McLendon, J. Phys. Chem. 90, 2549 (1986).

${ }^{10}$ Yu. T. Mazurenko and N. G. Bakhshiev, Opt. Spectrosc. 28, 490 (1970).

${ }^{11}$ A. I. Burshtein and A. G. Kofman, Chem. Phys. 40, 289 (1979).

${ }^{12}$ L. D. Zusman, (a) Chem. Phys. 49, 295 (1980); (b) Chem. Phys. Lett. 86, 547 (1982); (c) Chem. Phys. 80, 29 (1983).

${ }^{13}$ I. V. Alexandrov, Chem. Phys. 51, 449 (1980).

${ }^{14}$ M. Ya. Ovchinnikova, Teor. Eksp. Khim. 17, 651 (1981) [English translation: Theor. Exp. Chem. 17, 507 (1982)].

${ }^{15}$ A. B. Helman, Chem. Phys. 65, 271 (1982).

${ }^{16}$ G. van der Zwan and J. T. Hynes, J. Chem. Phys. 76, 2993 (1982); J. Phys. Chem. 89, 4181 (1985).

${ }^{17}$ D. F. Calef and P. G. Wolynes, J. Phys. Chem. 87, 3387 (1983); J. Chem. Phys. 78, 470 (1983).

${ }^{18}$ H. Sumi and R. A. Marcus, J. Chem. Phys. 84, 4894 (1986) (part I).

${ }^{19}$ (a) H. Sumi and R. A. Marcus, J. Chem. Phys. 84, 4272 (1986); (b) R. A. Marcus and H. Sumi, J. Electroanal. Chem. 204, 59 (1986).

${ }^{20} \mathrm{~K}$. Schulten, A. Brünger, W. Nadler, and Z. Schulten, in SynergeticsFrom Microscopic to Macroscopic Order, edited by E. Frehland (Springer, Berlin, 1984), pp. 80-89. There is a typographical error in Eq. (20) where a should be replaced by $a^{N}$.

${ }^{21}$ W. Nadler and K. Schulten, J. Chem. Phys. 84, 4015 (1986).

${ }^{22}$ (a) This assumption enters in the preexponential factor $v_{q}$ in the expression for $k(X)$, Eq. (2.6). (b) This reactive term is, in effect, the transition state theory expression for the reaction rate at a certain value of the polarization coordinate $X$. The exponential factor in $\mathrm{Eq} .(2.6)$ is the Boltzmann probability that a coordinate $q$, which represents the totality of the intramolecular coordinates, has the transition state value if the system sees a specified value of the polarization coordinate $X$. Hereby the assumption enters that during the reaction the distribution of states along a $q$ hypersurface of the $(X, q)$ space on the reactant side of the transition state line is a Boltzmann distribution. This is achieved through the "fast" equilibration in the $q$ coordinate. A picture of the corresponding free energy surface for the reaction is given in Fig. 1 of part I. The pre-factor $v_{q}$ in Eq. (2.6) is large in the case that the reaction is adiabatic, i.e., in the case that it proceeds with a large probability on the lowest potential energy surface. Otherwise, $v_{q}$ is small. The adiabatic and nonadiabatic expressions for $v_{q}$ are given in part I, Eqs. (4.9) and (4.11). (c) An exception is the situation of an adiabatic reaction in the limiting case of a vanishing contribution from the intramolecular coordinates $q$. For $\lambda_{i} / \lambda_{0} \rightarrow 0$ the transition state line gradually approaches the line $X=X_{c}$ which is perpendicular to the $X$ axis. This leads to a delta-function form of the reactive term as discussed later in the text in more detail. If the reaction is totally adiabatic the reactant molecules can only be on the reactant side of the transition state line and, therefore, the initial distribution $P(X, t=0)$ can be nonzero only for values of $X<X_{c}$. See also Fig. 1 in part I. Such a situation corresponds to the models discussed in Refs. 13 and 17.

${ }^{23} \mathrm{An}$ introduction to mean first passage time approaches can be found in modern textbooks on stochastic processes, e.g., (a) N. G. van Kampen, Stochastic Processes in Physics and Chemistry (North-Holland, Amsterdam, 1981); (b) C. W. Gardiner, Handbook of Stochastic Methods (Springer, Berlin, 1983); (c) H. Risken, The Fokker-Planck Equation (Springer, Berlin, 1984).

${ }^{24}$ K. Schulten, Z. Schulten, and A. Szabo, Physica A 100, 599 (1980).

${ }^{25}$ See also the discussion of the results of Ref. 11 concerning this case in part I.

${ }^{26} \mathrm{~A}$ rather general review of reaction diffusion models and their treatment has been published recently by G. H. Weiss, J. Stat. Phys. 42, 3 (1986).

${ }^{27}$ H. A. Kramers, Physica 7, 284 (1940).

${ }^{28}$ A similar set of equations has been derived in Refs. 12(b) and 14. Resulting reaction rates have been calculated approximately, for certain limiting cases, in Refs. 14 and 12(c).

${ }^{29}$ (a) N. Agmon and J. J. Hopfield, J. Chem. Phys. 78, 6947 (1983); (b) 79, 2042 (1983).

${ }^{30}$ B. Bagchi, G. R. Fleming, and D. W. Oxtoby, J. Chem. Phys. 78, 7375 (1983). 
${ }^{31}$ This scalar product is closely related to the thermal equilibrium expectation value $\langle U V\rangle_{0}$, compare Eq. (2.8). However, in case of the scalar product attention has to be paid to the correct ordering of the functions under the integral because of the action of any operator between $U$ and $V$ on $V$.

${ }^{32}$ G. A. Baker and P. Graves-Morris, Padé Approximants I, II (AddisonWesley, Reading, Mass., 1981).

${ }^{33}$ A. Brünger, R. Peters, and K. Schulten, J. Chem. Phys. 82, 2147 (1985).

${ }^{34}$ This symmetrization of the Fokker-Planck operator is now a standard technique, see, e.g., Ref. 23(c).

${ }^{35}$ W. H. Press, B. P. Flannery, S. A. Teukolsky, and W. T. Vetterling, $\mathrm{Nu}$ merical Recipes (Cambridge University, Cambridge, 1986).

${ }^{36}$ This disagreement is mainly due to the fact that the expressions in Eqs. (7.8) and (7.9) of part I do not ultimately approach the correct form in the slow diffusion limit, $\tau_{L} k_{e} \rightarrow \infty$.

${ }^{37} \mathrm{~W}$. Nadler (in preparation).

${ }^{38}$ G. H. Weiss, J. Chem. Phys. 80, 2880 (1984).

${ }^{39}$ G. Wilemski and M. Fixman, J. Chem. Phys. 58, 4009 (1973). These authors derive a Lippmann-Schwinger equation for $Q(t)$ and approximate the distribution $P(X, t)$ in the interaction term by $P_{0}(X) Q(t)$. Using this result, in Ref. 38 the correction Eq. (3.3a) to the fast diffusion limit of the average survival time $\tau_{a}$ is derived, with the matrix element given by Eq. (B9).

${ }^{40} \mathrm{Hereby}$ we have neglected the contribution from $I_{1}(0)$ and have approximated the error function in the integrand of $F_{1}$, Eq. (A9a), by unity since the main contributions to the integral come from the large values of the exponential function in the integrand.

${ }^{41}$ Handbook of Mathematical Functions, edited by M. Abramowitz and I. A. Stegun, Natl. Bur. Stand. (U. S. GPO, Washington, D. C., 1972), p. 319.

${ }^{42}$ R. K. Huddleston and J. Miller, J. Phys. Chem. 86, 200 (1982), and references cited therein.

${ }^{43}$ H. Frauenfelder and P. G. Wolynes, Science 229, 337 (1985), and references cited therein; see also Ref. 29.

${ }^{44}$ Related references to this literature are given in Ref. 19(a).

${ }^{45}$ See Ref. 9 and references cited therein.

${ }^{46}$ See upper curve in Fig. 2 of Ref. 9.

${ }^{47}$ A. Szabo, K. Schulten, and Z. Schulten, J. Chem. Phys. 72, 4350 (1980).

${ }^{48}$ In Ref. 24 a trick to calculate at least Eq. (A7a) directly from Eq. (A6) is shown.

${ }^{49}$ W. Nadler and K. Schulten, J. Chem. Phys 82, 151 (1985) 